TITLE:

\title{
Variational calculations of fermion second-order reduced density matrices by semidefinite programming algorithm
}

\section{$\operatorname{AUTHOR(S):~}$}

Nakata, M; Nakatsuji, H; Ehara, M; Fukuda, M; Nakata, K; Fujisawa, K

\section{CITATION:}

Nakata, M ...[et al]. Variational calculations of fermion second-order reduced density matrices by semidefinite programming algorithm. JOURNAL OF CHEMICAL PHYSICS 2001, 114(19): 8282-8292

\section{ISSUE DATE:}

2001-05-15

URL:

http://hdl.handle.net/2433/39729

\section{RIGHT:}

Copyright 2001 American Institute of Physics. This article may be downloaded for personal use only. Any other use requires prior permission of the author and the American Institute of Physics. 


\title{
Variational calculations of fermion second-order reduced density matrices by semidefinite programming algorithm
}

\author{
Maho Nakata, Hiroshi Nakatsuji, a) and Masahiro Ehara \\ Department of Synthetic Chemistry and Biological Chemistry, Graduate School of Engineering, \\ Kyoto University, Kyoto 606-8501, Japan \\ Mitsuhiro Fukuda \\ Department of Mathematical and Computing Sciences, Tokyo Institute of Technology, \\ Tokyo 152-8852, Japan \\ Kazuhide Nakata \\ Department of Applied Physics, The University of Tokyo, Tokyo 113-8656, Japan \\ Katsuki Fujisawa \\ Department of Architecture and Architectural Systems, Kyoto University, Kyoto 606-8501, Japan
}

(Received 24 July 2000; accepted 9 February 2001)

\begin{abstract}
The ground-state fermion second-order reduced density matrix (2-RDM) is determined variationally using itself as a basic variable. As necessary conditions of the $N$-representability, we used the positive semidefiniteness conditions, $P, Q$, and $G$ conditions that are described in terms of the 2-RDM. The variational calculations are performed by using recently developed semidefinite programming algorithm (SDPA). The calculated energies of various closed- and open-shell atoms and molecules are excellent, overshooting only slightly the full-CI energies. There was no case where convergence was not achieved. The calculated properties also reproduce well the full-CI results. (ㅇ) 2001 American Institute of Physics. [DOI: 10.1063/1.1360199]
\end{abstract}

\section{INTRODUCTION}

The ground state of $N$-body fermion system is completely described by the second-order reduced density matrix $(2-R D M) \Gamma^{(2)}$ because any observable properties of the system can be calculated from the 2-RDM. ${ }^{1,2}$ This fact led us to desire to use 2-RDM as a basic variable of quantum mechanics instead of the wave function $\Psi$; if we can determine $\Gamma^{(2)}$ without using $\Psi$, we have a closed form of quantum mechanics where the basic variable is 2-RDM. We refer to such formalism of quantum mechanics as density matrix theory (DMT). ${ }^{3}$

In the nonrelativistic case, the determinative equation for $\Psi$ is the Schrödinger equation (SE). Therefore, to establish DMT, we have to formulate the equation for the RDM that is equivalent to the SE in the necessary and sufficient sense. ${ }^{3}$ As such an equation, one of the author derived density equation (DE) (Refs. 4 and 5) that has recently been used successfully to calculate the 2-RDMs of atoms and molecules directly without any use of the wave function. ${ }^{6-9}$ This approach is called density equation theory (DET) and a review on the DET in chemical physics has recently been summarized together with some later developments. ${ }^{3}$

Another equation that is equivalent to the SE but includes $2-\mathrm{RDM}$ alone as a variable is the variational equation of the form,

$$
E_{g} \leqslant E\left[\Gamma^{(2)}\right],
$$

where $E_{g}$ is the exact ground-state energy. This method called density matrix variational theory (DMVT) is a

${ }^{a)}$ Author to whom correspondence should be addressed. straightforward consequence of the Ritz variational principle combined with the fact that the Hamiltonian involves only one- and two-body operators. The problem here is how well we can restrict our variable $\Gamma^{(2)}$ to be $N$-representable. ${ }^{10}$ The $N$-representability condition that is enforced by the Pauli principle is not completely known for $\Gamma^{(2)}$ and this is an obstacle of the DMT in general.

The $P, Q,($ Ref. 10) and $G$ (Ref. 11) conditions are the well-known necessary conditions of the $N$-representability. They are the semidefiniteness conditions of the matrices derived from $\Gamma^{(2)}$. Though these three conditions are not complete, they seem to be quite strong to characterize the $\mathrm{N}$-representability of the ground-state 2-RDM. First calculations along this line were performed in a beautiful way by Garrod et al. ${ }^{12,13}$ for the ground state of $\mathrm{Be}$, and Mihailović et al. ${ }^{14}$ for the nuclear ground state of ${ }^{15} \mathrm{O},{ }^{16} \mathrm{O},{ }^{17} \mathrm{O},{ }^{18} \mathrm{O}$, ${ }^{20} \mathrm{Ne},{ }^{24} \mathrm{Mg}$, and ${ }^{28} \mathrm{Si}$. At that time their method was very heuristic and could hardly be applied to general systems. We found that this method can be elegantly realized using the semidefinite programming algorithm (SDPA), ${ }^{15}$ recently developed in the field of mathematical programming. We calculated the ground-state energies of atoms and molecules using these three necessary conditions and employing SDPA as our problem solver.

\section{THEORICAL OUTLINE}

First and second order reduced density matrices (1-, 2-RDMs), $\gamma$ and $\Gamma$, respectively, are defined by

$$
\gamma_{j}^{i}=\left\langle\Psi\left|a_{i}^{\dagger} a_{j}\right| \Psi\right\rangle
$$

and 


$$
\Gamma_{j_{1} j_{2}}^{i_{1} i_{2}}=\frac{1}{2}\left\langle\Psi\left|a_{i_{1}}^{\dagger} a_{i_{2}}^{\dagger} a_{j_{2}} a_{j_{1}}\right| \Psi\right\rangle
$$

where $a^{\dagger}$ and $a$ denote creation and annihilation operators, respectively. Note we have simplified $\Gamma^{(2)}$ as $\Gamma$. Throughout this paper, we assume the elements of 1-RDM and 2-RDM to be real. Complete $N$-representability condition is known for $\gamma,{ }^{10}$ but for $\Gamma$, we know only necessary conditions (the known complete condition is not practical). Some trivial conditions for 2-RDM are:

(1) Antisymmetric condition,

$\Gamma_{j_{1} j_{2}}^{i_{1} i_{2}}=-\Gamma_{j_{1} j_{2}}^{i_{2} i_{1}}=-\Gamma_{j_{2} j_{1}}^{i_{1} i_{2}}$

(2) Hermiticity,

$\Gamma_{j_{1} j_{2}}^{i_{1} i_{2}}=\Gamma_{i_{1} i_{2}}^{j_{1} j_{2}}$

(3) Trace condition,

$$
\sum_{k} \Gamma_{j k}^{i k}=\frac{2}{N-1} \gamma_{j}^{i} ;
$$

(4) Number of electrons,

$$
N=\sum_{k} \gamma_{k}^{k}
$$

(5) Eigenstate of the number of $\alpha$ (or $\beta$ ) electrons,

$\operatorname{Tr} N_{\alpha} \Gamma=N_{\alpha}$ and $\operatorname{Tr} N_{\alpha}^{2} \Gamma=N_{\alpha}^{2} ;$

where the operators of $N_{\alpha}$ and $N_{\alpha}^{2}$ are written as

$$
N_{\alpha}=\sum_{i} a_{i \alpha}^{\dagger} a_{i \alpha}, \quad N_{\alpha}^{2}=\sum_{i j} a_{i \alpha}^{\dagger} a_{i \alpha} a_{j \alpha}^{\dagger} a_{j \alpha}
$$

(6) Spin symmetry,

$$
\Gamma_{j_{1} \sigma_{1}^{\prime} j_{2} \sigma_{2}^{\prime}}^{i_{1} \sigma_{1} i_{2} \sigma_{2}}=0
$$

when $\sigma_{1} \neq \sigma_{1}^{\prime}$ or $\sigma_{2} \neq \sigma_{2}^{\prime}$ and $\sigma_{1} \neq \sigma_{2}^{\prime}$ or $\sigma_{2} \neq \sigma_{1}^{\prime}$, where $\sigma$ denotes spin variable.

(7) Expectation value of $S^{2}$

$$
\operatorname{Tr} S^{2} \Gamma=S(S+1),
$$

where the spin-squared operator $S^{2}$ is given by

$$
\begin{aligned}
S^{2}= & S_{z}+S_{z}^{2}+S_{-} S_{+} \\
= & \frac{1}{2} \sum_{i}\left(a_{i \alpha}^{\dagger} a_{i \alpha}-a_{i \beta}^{\dagger} a_{i \beta}\right)+\frac{1}{4}\left(\sum_{i} a_{i \alpha}^{\dagger} a_{i \alpha}-a_{i \beta}^{\dagger} a_{i \beta}\right)^{2} \\
& +\sum_{i j} a_{i \beta}^{\dagger} a_{i \alpha} a_{j \alpha}^{\dagger} a_{j \beta} ;
\end{aligned}
$$

(8) Positive semidefiniteness of $P$ matrix, which is just 2-RDM,

$$
\sum x_{i_{1} i_{2}} \Gamma_{j_{1} j_{2}}^{i_{1} i_{2}} x_{j_{1} j_{2}} \geqslant 0
$$

where $x_{i_{1} i_{2}}$ is an arbitrary geminal.

Note that except for the condition 8 , all of these conditions are linear to 2-RDM.

The $Q$ and $G$ matrices are defined by

$$
Q_{j_{1} j_{2}}^{i_{1} i_{2}}=\left\langle\Psi\left|a_{i_{1}} a_{i_{2}} a_{j_{2}}^{\dagger} a_{j_{1}}^{\dagger}\right| \Psi\right\rangle
$$

and

$$
G_{j_{1} j_{2}}^{i_{1} i_{2}}=\left\langle\Psi\left|a_{i_{1}}^{\dagger} a_{i_{2}} a_{j_{2}}^{\dagger} a_{j_{1}}\right| \Psi\right\rangle .
$$

These matrices are semidefinite ${ }^{11}$ and linear to $\Gamma$ as

$$
\begin{aligned}
Q_{j_{1} j_{2}}^{i_{1} i_{2}}= & \left(\delta_{j_{1}}^{i_{1}} \delta_{j_{2}}^{i_{2}}-\delta_{j_{2}}^{i_{1}} \delta_{j_{1}}^{i_{2}}\right)-\left(\delta_{j_{1}}^{i_{1}} \gamma_{j_{2}}^{i_{2}}+\delta_{j_{2}}^{i_{2}} \gamma_{j_{1}}^{i_{1}}\right) \\
& +\left(\delta_{j_{2}}^{i_{1}} \gamma_{j_{1}}^{i_{2}}+\delta_{j_{1}}^{i_{2}} \gamma_{j_{2}}^{i_{1}}\right)-2 \Gamma_{j_{1} j_{2}}^{i_{1} i_{2}}
\end{aligned}
$$

and

$$
G_{j_{1} j_{2}}^{i_{1} i_{2}}=\delta_{j_{2}}^{i_{2}} \gamma_{j_{1}}^{i_{1}}-2 \Gamma_{j_{1} i_{2}}^{i_{1} j_{2}}
$$

We note that originally the $G$ matrix was written in an equivalent nonlinear form. ${ }^{16}$

The Hamiltonian of the system can be written as

$$
H_{j_{1} j_{2}}^{i_{1} i_{2}}=w_{j_{1} j_{2}}^{i_{1} i_{2}}+\frac{1}{N-1}\left(v_{j_{1}}^{i_{1}} \delta_{j_{2}}^{i_{2}}+v_{j_{2}}^{i_{1}} \delta_{j_{1}}^{i_{2}}\right)
$$

where $v$ and $w$ are 1- and 2-body operators, respectively. Then, the basic equation of DMVT given by Eq. (1.1) is written as the variational minimization of the energy within our constraints,

$$
E_{\min }=\min _{\Gamma \in{ }^{(2)} \mathcal{P}} \operatorname{Tr} H \Gamma,
$$

where ${ }^{(2)} \mathcal{P}$ is the set of 2 -RDMs which satisfies the above necessary $N$-representability conditions, namely,

${ }^{(2)} \mathcal{P}=\{\Gamma \mid P, Q, G$ matrices are non-negative and the

$$
\text { conditions } 1-7 \text { are satisfied\}. }
$$

Either of the $P, Q$, and $G$ conditions forms compact convex set with trace topology, ${ }^{17}$ and a finite combination of compact convex sets is also compact convex set, therefore this method should find a minimum in energy. This method can be applied to the ground state of any space and spin symmetry.

\section{CALCULATION METHOD}

The minimization problem with some linear constraints can be achieved by using semidefinite programming algorithm (SDPA) (Ref. 15) as a problem solver. The SDPA has recently been developed in the field of mathematical programming. In this section, we explain how to apply SDPA to our problem of solving $\Gamma^{(2)}$ in the constrained variational method given by Eq. (2.19). The dimensions of the matrices are $n \times n$, if they are not explicitly defined.

\section{A. Simplified problem}

First, we introduce a simplified problem which contains all the essentials, that is

Problem (a): Minimize the total energy of the 2-RDM $\Gamma$ subject to the fixed number of electrons and the positive semidefiniteness of $\Gamma$.

The positive semidefiniteness of $\Gamma$ is the $P$ condition. Note that this problem gives the exact solution for $N=2$. Problem (a) is written as, 


$$
\text { Problem }\left(\mathrm{a}^{\prime}\right):\left\{\begin{array}{l}
\text { Minimize } \operatorname{Tr} H \Gamma \\
\text { subject to } \operatorname{Tr} N \Gamma=N \\
\text { and } \Gamma \text { is positive semidefinite. }
\end{array}\right.
$$

Formal expression of the problem ${ }^{15}$ is,

$$
\text { Problem }\left(\mathrm{a}^{\prime \prime}\right):\left\{\begin{array}{l}
\text { Minimize } \mathbf{F}_{0} \cdot \mathbf{Y} \\
\text { subject to } \mathbf{F}_{1} \cdot \mathbf{Y}=c_{1} \\
\text { and } \mathbf{Y} \text { is positive semidefinite, }
\end{array}\right.
$$

where $\mathbf{F}_{0}$ and $\mathbf{F}_{1}$ are constant $n \times n$ symmetric matrices, and $\mathbf{Y}$ is $n \times n$ symmetric variable matrix, $c_{1}$ is real constant, and - is an operator such that

$$
\mathbf{F} \cdot \mathbf{Y}=\sum_{i, j}(\mathbf{F})_{i j}(\mathbf{Y})_{i j}
$$

One can easily confirm that problem $\left(a^{\prime}\right)$ and problem $\left(a^{\prime \prime}\right)$ are the same when we take $\mathbf{Y}$ as $\Gamma, \mathbf{F}_{0}$ as the Hamiltonian, and $\mathbf{F}_{1}$ as the number of operator. A generalization of the problem $\left(a^{\prime \prime}\right)$ is called semidefinite programming (SDP).

\section{B. Semidefinite programming algorithm (SDPA)}

The SDPA (Ref. 15) solves the following form of semidefinite programming and its dual:

$$
\operatorname{SDP} \begin{cases}\text { primal: } & \text { minimize } \sum_{i=1}^{m} c_{i} x_{i} \\ & \text { subject to } \mathbf{X}=\sum_{i=1}^{m} \mathbf{F}_{i} x_{i}-\mathbf{F}_{0}, \quad \mathbf{X} \geqslant \mathbf{0} \\ \text { dual: } \quad \text { maximize } \mathbf{F}_{0} \cdot \mathbf{Y} & \text { subject to } \mathbf{F}_{i} \cdot \mathbf{Y}=c_{i}(1 \leqslant i \leqslant m), \quad \mathbf{Y} \geqslant \mathbf{0},\end{cases}
$$

where $\mathbf{X}$ and $\mathbf{Y}$ are $n \times n$ real symmetric matrices, $\mathbf{F}_{i}(1 \leqslant i$ $\leqslant m)$ symmetric constraint matrices, $c_{i}$ and $x_{i}$ real constant and variable numbers, respectively, $\mathbf{U} \cdot \mathbf{V}$ denotes inner product of the matrices, $\mathbf{U} \cdot \mathbf{V}=\Sigma_{i, j} \mathbf{U}_{i, j} \mathbf{V}_{i, j}$, and $\mathbf{X} \geqslant \mathbf{0}$ means $\mathbf{X}$ to be positive semidefinite. We assume all the constraint matrices are linearly independent.

Semidefinite programming is usually solved by primaldual interior-point method. ${ }^{18,19}$ This method is based on the primal-dual theorem of SDP, which shows an existence of the optimal solution and gives a necessary and sufficient condition for the optimal solution (minimum in primal problem, and maximum in dual problem): if there exists $(\mathbf{X}, \mathbf{Y}, \mathbf{x})$ such that they satisfy all the constraints and $\mathbf{X} \geqslant \mathbf{0}$ and $\mathbf{Y} \geqslant \mathbf{0}$, then

(1) SDP has an optimal solution;

(2) Necessary and sufficient condition for the optimal solution $\left(\mathbf{X}^{*}, \mathbf{Y}^{*}, \mathbf{x}^{*}\right)$ is

$$
\mathbf{X}^{*} \cdot \mathbf{Y}^{*}=\mathbf{F}_{0} \cdot \mathbf{Y}^{*}-\sum_{i=1}^{m} c_{i} x_{i}^{*}=0 .
$$

\section{Set up of DMVT in SDPA}

Our object is to solve the DMVT problem (2.19). It is equivalent to solve the dual of problem (3.4), taking $\mathbf{Y}$ as 2-RDM, $\mathbf{F}_{0}$ as the Hamiltonian, $\mathbf{F}_{1}$ as the constraint for the number of electrons, $\mathbf{F}_{2}$ as the constraint for spin squared operator, etc. Maximization is altered to minimization by just changing the sign of $\mathbf{F}_{0}$. Problem (2.19) is written as

Minimize $\operatorname{Tr} H \Gamma$

$$
\begin{aligned}
& \text { subjected to } \operatorname{Tr} N \Gamma=N \\
& \qquad \begin{array}{lr}
\operatorname{Tr} S^{2} \Gamma=S(S+1) \\
& \operatorname{Tr} N_{\alpha} \Gamma=N_{\alpha} \\
& \operatorname{Tr} N_{\alpha}^{2} \Gamma=N_{\alpha}^{2} \\
& \text { and } \Gamma^{(2)} \geqslant 0, \quad \mathbf{Q} \geqslant 0 \text { and } \mathbf{G} \geqslant 0 .
\end{array}
\end{aligned}
$$

Note that some of the matrices appeared below have four indices, however, we can reduce them to two indices by mapping indices $(i, j)$ to the composite index $k$. Imposing linear constraints for $N$ or $S^{2}$, etc., is straightforward. Constraining the expectation value of the two body operator $A$ to be $c_{a}\left(\operatorname{Tr} A \Gamma=c_{a}\right)$ is done as follows:

(1) Explicit expression of $A$ is give by

$$
A=\sum_{i_{1} i_{2} j_{1} j_{2}} a_{j_{1} j_{2}}^{i_{1} i_{2}} a_{i_{1}}^{\dagger} a_{i_{2}}^{\dagger} a_{j_{2}} a_{j_{1}}
$$

where $a_{j_{1} j_{2}}^{i_{1} i_{2}}$ is constant.

(2) Set up the constraint matrix $\mathbf{F}_{A}$ such that

$\left(\mathbf{F}_{A}\right)_{j_{1} j_{2}}^{i_{1} i_{2}}=a_{j_{1} j_{2}}^{i_{1} i_{2}}$

(3) Then, the constraint is given by the equality,

$$
\mathbf{F}_{A} \cdot \Gamma=\sum_{i_{1} i_{2} j_{1} j_{2}} a_{j_{1} j_{2}}^{i_{1} i_{2}} Y_{j_{1} j_{2}}^{i_{1} i_{2}}=\operatorname{Tr} A \Gamma=c_{a}
$$

For example, we set up the constraint matrix for the number of particles $N$. Explicit expression of $N$ is,

$$
N=\sum_{i} a_{i}^{\dagger} a_{i}=\frac{N-1}{2} \sum_{i j} a_{i}^{\dagger} a_{j}^{\dagger} a_{j} a_{i} .
$$

Then, the constraint matrix $\mathbf{F}_{N}$ for the number of particle is represented by

$$
\left(\mathbf{F}_{N}\right)_{k l}^{i j}=\frac{N-1}{2} \delta_{i k} \delta_{j l},
$$

and we confirm the following relation:

$$
\begin{aligned}
\mathbf{F}_{N} \cdot \mathbf{Y} & =\sum_{i j k l}\left(\mathbf{F}_{N}\right)_{k l}^{i j} \Gamma_{k l}^{i j} \\
& =\sum_{i j k l} \frac{N-1}{2} \delta_{i k} \delta_{j l} \Gamma_{k l}^{i j} \\
& =\frac{N-1}{2} \sum_{i j} \Gamma_{i j}^{i j} \\
& =\frac{N-1}{2} \sum_{i} \frac{2}{N-1} \gamma_{i}^{i}=\sum_{i} \gamma_{i}^{i}=N .
\end{aligned}
$$

Now we consider how to enforce the 2-RDM to satisfy the $P, Q$, and $G$ conditions, simultaneously. We first explain the case where only $P$ and $Q$ conditions are enforced simultaneously. 
We introduce the variable matrix $\mathbf{Y}$ in which $P$ and $Q$ matrices are diagonally arranged,

$$
\mathbf{Y}=\left(\begin{array}{ll}
\mathbf{P} & \mathbf{0} \\
\mathbf{0} & \mathbf{Q}
\end{array}\right) \text {. }
$$

It is obvious that

$$
\mathbf{Y} \geqslant \mathbf{0} \leftrightarrow \mathbf{P} \geqslant \mathbf{0} \text { and } \mathbf{Q} \geqslant \mathbf{0} .
$$

There is a linear relation between $\Gamma$ and $Q$ matrices,

$$
\begin{aligned}
Q_{j_{1} j_{2}}^{i_{1} i_{2}}= & \left(\delta_{j_{1}}^{i_{1}} \delta_{j_{2}}^{i_{2}}-\delta_{j_{2}}^{i_{1}} \delta_{j_{1}}^{i_{2}}\right)-\sum_{k} \frac{N-1}{2}\left(\delta_{j_{1}}^{i_{1}} \Gamma_{j_{2} k}^{i_{2} k}+\delta_{j_{2}}^{i_{2}} \Gamma_{j_{1} k}^{i_{1} k}\right) \\
& +\sum_{k} \frac{N-1}{2}\left(\delta_{j_{2}}^{i_{1}} \Gamma_{j_{1} k}^{i_{2} k}+\delta_{j_{1}}^{i_{2}} \Gamma_{j_{2} k}^{i_{1} k}\right)-2 \Gamma_{j_{1} j_{2}}^{i_{1} i_{2}} .
\end{aligned}
$$

Therefore, we can find a set of linear constraints for each element of the $Q$ matrix as

$$
\begin{aligned}
\mathbf{E}_{j_{1} j_{2}}^{i_{1} i_{2}} \cdot \mathbf{Y}=c_{j_{1} j_{2}}^{i_{1} i_{2}}= & 2 \Gamma_{j_{1} j_{2}}^{i_{1} i_{2}}+\left(\delta_{j_{1}}^{i_{1}} \gamma_{j_{2}}^{i_{2}}+\delta_{j_{2}}^{i_{2}} \gamma_{j_{1}}^{i_{1}}\right) \\
& -\left(\delta_{j_{2}}^{i_{1}} \gamma_{j_{1}}^{i_{2}}+\delta_{j_{1}}^{i_{2}} \gamma_{j_{2}}^{i_{1}}\right)+Q_{j_{1} j_{2}}^{i_{1} i_{2}} \\
= & \delta_{j_{1}}^{i_{1}} \delta_{j_{2}}^{i_{2}}-\delta_{j_{2}}^{i_{1}} \delta_{j_{1}}^{i_{2}} .
\end{aligned}
$$

Using these constraints, the SDP formalism is given by

\section{Minimize $\mathbf{H} \cdot \mathbf{Y}$}

subject to $\mathbf{F}_{i} \cdot \mathbf{Y}=c_{i}$

$$
\widetilde{\mathbf{E}}_{j_{1} j_{2}}^{i_{1} i_{2}} \cdot \mathbf{Y}=\delta_{j_{1}}^{i_{1}} \delta_{j_{2}}^{i_{2}}-\delta_{j_{2}}^{i_{1}} \delta_{j_{1}}^{i_{2}}
$$

where $\widetilde{\mathbf{E}}_{j_{1} j_{2}}^{i_{1} i_{2}}$ is a symmetric matrix defined by

$$
\widetilde{\mathbf{E}}_{j_{1} j_{2}}^{i_{1} i_{2}}=\frac{1}{2}\left(\mathbf{E}_{j_{1} j_{2}}^{i_{1} i_{2}}+\mathbf{E}_{i_{1} i_{2}}^{j_{1} j_{2}}\right),
$$

and the explicit expression of the element of the constraint matrix $\left(\mathbf{E}_{j_{1} j_{2}}^{i_{1} i_{2}}\right)_{l_{1} l_{2}}^{k_{1} k_{2}}$ is given by

$$
\begin{aligned}
\left(\mathbf{E}_{j_{1} j_{2}}^{i_{1} i_{2}}\right)_{l_{1} l_{2}}^{k_{1} k_{2}}= & 2 \delta_{k_{1}}^{i_{1}} \delta_{k_{2}}^{i_{2}} \delta_{l_{1}}^{j_{1}} \delta_{l_{2}}^{j_{2}}+\delta_{k_{1}}^{i_{1}+n} \delta_{k_{2}}^{i_{2}+n} \delta_{l_{1}}^{j_{1}+n} \delta_{l_{2}}^{j_{2}+n} \\
& +\frac{N-1}{2} \delta_{j_{1}}^{i_{1}} \delta_{k_{1}}^{i_{2}} \delta_{l_{1}}^{j_{2}} \delta_{l_{2}}^{k_{2}}+\frac{N-1}{2} \delta_{j_{2}}^{i_{2}} \delta_{k_{1}}^{i_{1}} \delta_{l_{1}}^{j_{1}} \delta_{l_{2}}^{k_{2}} \\
& -\frac{N-1}{2} \delta_{j_{2}}^{i_{1}} \delta_{k_{1}}^{i_{2}} \delta_{l_{1}}^{j_{1}} \delta_{l_{2}}^{k_{2}}-\frac{N-1}{2} \delta_{j_{1}}^{i_{2}} \delta_{k_{1}}^{i_{1}} \delta_{l_{1}}^{j_{2}} \delta_{l_{2}}^{k_{2}}
\end{aligned}
$$

and the constant $c_{j_{1} j_{2}}^{i_{1} i_{2}}$ in Eq. (3.16) is

$$
c_{j_{1} j_{2}}^{i_{1} i_{2}}=\delta_{j_{1}}^{i_{1}} \delta_{j_{2}}^{i_{2}}-\delta_{j_{2}}^{i_{1}} \delta_{j_{1}}^{i_{2}}
$$

We can confirm Eq. (3.21) holds

$$
\mathbf{E}_{j_{1} j_{2}}^{i_{1} i_{2}} \cdot \mathbf{Y}=\sum_{k_{1} k_{2} l_{1} l_{2}}\left(\mathbf{E}_{j_{1} j_{2}}^{i_{1} i_{2}}\right)_{l_{1} l_{2}}^{k_{1} k_{2}}(\mathbf{Y})_{l_{1} l_{2}}^{k_{1} k_{2}}=c_{j_{1} j_{2}}^{i_{1} i_{2}}
$$

as follows. The first two terms of Eq. (3.21) are

$$
\begin{gathered}
\sum_{k_{1} k_{2} l_{1} l_{2}}\left(2 \delta_{k_{1}}^{i_{1}} \delta_{k_{2}}^{i_{2}} \delta_{l_{1}}^{j_{1}} \delta_{l_{2}}^{j_{2}}+\delta_{k_{1}}^{i_{1}+n} \delta_{k_{2}}^{i_{2}+n} \delta_{l_{1}}^{j_{1}+n} \delta_{l_{2}}^{j_{2}+n}\right)(\mathbf{Y})_{l_{1} l_{2}}^{k_{1} k_{2}} \\
=2(\mathbf{Y})_{j_{1} j_{2}}^{i_{1} i_{2}}+(\mathbf{Y})_{j_{1}+n, j_{2}+n}^{i_{1}+n, i_{2}+n}=2 \Gamma_{j_{1} j_{2}}^{i_{1} i_{2}}+Q_{j_{1} j_{2}}^{i_{1} i_{2}},
\end{gathered}
$$

the second two terms of Eq. (3.21) give,

$$
\begin{gathered}
\sum_{k_{1} k_{2} l_{1} l_{2}}\left(\frac{N-1}{2} \delta_{j_{1}}^{i_{1}} \delta_{k_{1}}^{i_{2}} \delta_{l_{1}}^{j_{2}} \delta_{l_{2}}^{k_{2}}+\frac{N-1}{2} \delta_{j_{2}}^{i_{2}} \delta_{k_{1}}^{i_{1}} \delta_{l_{1}}^{j_{1}} \delta_{l_{2}}^{k_{2}}\right)(\mathbf{Y})_{l_{1} l_{2}}^{k_{1} k_{2}} \\
=\frac{N-1}{2} \sum_{k} \delta_{j_{1}}^{i_{1}} \Gamma_{j_{2} k}^{i_{2} k}+\frac{N-1}{2} \sum_{k} \delta_{j_{2}}^{i_{2}} \Gamma_{j_{1} k}^{i_{1} k} \\
=\delta_{j_{1}}^{i_{1}} \gamma_{j_{2}}^{i_{2}}+\delta_{j_{2}}^{i_{2}} \gamma_{j_{1}}^{i_{1}}
\end{gathered}
$$

and the last term gives

$$
\begin{gathered}
\sum_{k_{1} k_{2} l_{1} l_{2}}\left(-\frac{N-1}{2} \delta_{i_{1}}^{j_{2}} \delta_{k_{1}}^{i_{2}} \delta_{l_{1}}^{j_{1}} \delta_{l_{2}}^{k_{2}}-\frac{N-1}{2} \delta_{i_{2}}^{j_{1}} \delta_{k_{1}}^{i_{1}} \delta_{l_{1}}^{j_{2}} \delta_{l_{2}}^{k_{2}}\right)(\mathbf{Y})_{l_{1} l_{2}}^{k_{1} k_{2}} \\
=-\frac{N-1}{2} \sum_{k} \delta_{i_{1}}^{j_{2}} \Gamma_{j_{1} k}^{i_{2} k}-\frac{N-1}{2} \sum_{k} \delta_{i_{2}}^{j_{1}} \Gamma_{j_{2} k}^{i_{1} k} \\
=-\delta_{j_{2}}^{i_{1}} \gamma_{j_{1}}^{i_{2}}-\delta_{j_{1}}^{i_{2}} \gamma_{j_{2}}^{i_{1}} .
\end{gathered}
$$

Combining Eq. (3.22) and Eq. (3.24), we get Eq. (3.16).

Constraining $P, Q$, and $G$ matrices to be positive semidefinite is done in essentially the same way as above. In this case, the variable matrix $\mathbf{Y}$ is defined as

$$
\mathbf{Y}=\left(\begin{array}{lll}
\mathbf{P} & \mathbf{0} & \mathbf{0} \\
\mathbf{0} & \mathbf{Q} & \mathbf{0} \\
\mathbf{0} & \mathbf{0} & \mathbf{G}
\end{array}\right)
$$

We have a linear relation between $\Gamma$ and $G$,

$$
G_{j_{1} j_{2}}^{i_{1} i_{2}}=\delta_{j_{2}}^{i_{2}} \sum_{k} \frac{N-1}{2} \Gamma_{j_{1} k}^{i_{1} k}-2 \Gamma_{j_{1} i_{2}}^{i_{1} j_{2}},
$$

which is described by a set of linear constraints $\mathbf{J}_{j_{1} j_{2}}^{i_{1} i_{2}}$ for each element of $G$ matrix as

$$
\mathbf{J}_{j_{1} j_{2}}^{i_{1} i_{2}} \cdot \mathbf{Y}=0=-\delta_{j_{2}}^{i_{2}} \gamma_{j_{1}}^{i_{1}}+2 \Gamma_{j_{1} i_{2}}^{i_{1} j_{2}}+G_{j_{1} i_{2}}^{i_{1} j_{2}},
$$

and an explicit expression of the constraint matrix $\left(\mathbf{J}_{j_{1} j_{2}}^{i_{1} i_{2}}\right)_{l_{1} l_{2}}^{k_{1} k_{2}}$ is given by

$$
\begin{aligned}
\left(\mathbf{J}_{j_{1} j_{2}}^{i_{1} i_{2}}\right)_{l_{1} l_{2}}^{k_{1} k_{2}=} & 2 \delta_{k_{1}}^{i_{1}} \delta_{k_{2}}^{i_{2}} \delta_{l_{1}}^{j_{1}} \delta_{l_{2}}^{j_{2}}+\delta_{k_{1}}^{i_{1}+2 n} \delta_{k_{2}}^{i_{2}+2 n} \delta_{l_{1}}^{j_{1}+2 n} \delta_{l_{2}}^{j_{2}+2 n} \\
& -\frac{N-1}{2} \delta_{j_{2}}^{i_{2}} \delta_{k_{1}}^{i_{1}} \delta_{l_{1}}^{i_{2}} \delta_{l_{2}}^{k_{2}}
\end{aligned}
$$

which is further symmetrized as

$$
\widetilde{\mathbf{J}}_{j_{1} j_{2}}^{i_{1} i_{2}}=\frac{1}{2}\left(\mathbf{J}_{j_{1} j_{2}}^{i_{1} i_{2}}+\mathbf{J}_{i_{1} i_{2}}^{j_{1} j_{2}}\right) \text {. }
$$

Thus, the DMVT using the $P, Q$, and $G$ conditions is formulated into SDPA as

\section{Minimize $\mathbf{H} \cdot \mathbf{Y}$}

subjected to $\mathbf{F}_{i} \cdot \mathbf{Y}=c_{i}$

$$
\begin{aligned}
& \widetilde{\mathbf{E}}_{j_{1} j_{2}}^{i_{1} i_{2}} \cdot \mathbf{Y}=\delta_{j_{1}}^{i_{1}} \delta_{j_{2}}^{i_{2}}-\delta_{j_{2}}^{i_{1}} \delta_{j_{1}}^{i_{2}} \\
& \widetilde{\mathbf{J}}_{j_{1} j_{2} i_{2}}^{i_{2}} \cdot \mathbf{Y}=0 .
\end{aligned}
$$

It is convenient to fold our 2-RDM into a compact form,

$$
P_{j_{1} j_{2}}^{i_{1} i_{2}} \rightarrow P_{j}^{i}
$$


TABLE I. Total energy and correlation energy in $\%$ in parentheses calculated by the DMVT with $P+Q$ and $P+Q+G$ conditions compared with those obatined by the wave function methods, full CI, and Hartree-Fock. The basis set is STO-6G except for notice.

\begin{tabular}{|c|c|c|c|c|c|c|c|}
\hline System & State & $\begin{array}{c}\text { Active } \\
\mathrm{MO}^{\mathrm{a}}\end{array}$ & $\operatorname{Ele}(\alpha+\beta)^{\mathrm{b}}$ & $\mathrm{DM}(P+Q)$ & $\mathrm{DM}(P+Q+G)$ & Full CI & Hartree-Fock \\
\hline $\mathrm{Be}^{\mathrm{c}}$ & ${ }^{1} S$ & 4 & $4(2+2)$ & $-14.5934(176)$ & $-14.5827(100)$ & $-14.5827(100)$ & $-14.5685(0)$ \\
\hline $\mathrm{Be}$ & ${ }^{1} S$ & 5 & $4(2+2)$ & $-14.5579(103)$ & $-14.5561(100)$ & $-14.5561(100)$ & $-14.5034(0)$ \\
\hline $\mathrm{Be}^{\mathrm{d}}$ & ${ }^{1} S$ & 5 & $4(2+2)$ & $-14.6064(200)$ & $-14.5895(100)$ & $-14.5895(100)$ & $-14.5725(0)$ \\
\hline $\mathrm{Be}^{\mathrm{c}}$ & ${ }^{3} S$ & 4 & $4(3+1)$ & $-13.3168(120)$ & $-13.3146(100)$ & $-13.3146(100)$ & $-13.3036(0)$ \\
\hline $\mathrm{Be}^{\mathrm{d}}$ & ${ }^{3} S$ & 5 & $4(3+1)$ & $-14.3346(177)$ & $-14.3241(100)$ & $-14.3241(100)$ & $-14.3105(0)$ \\
\hline $\mathrm{LiH}^{\mathrm{c}}$ & ${ }^{1} \Sigma^{+}$ & 6 & $4(2+2)$ & $-8.0034(139)$ & $-7.9924(100)$ & $-7.9922(100)$ & $-7.9635(0)$ \\
\hline $\mathrm{LiH}$ & ${ }^{1} \Sigma^{+}$ & 6 & $4(2+2)$ & $-7.9731(104)$ & $-7.9724(100)$ & $-7.9723(100)$ & $-7.9519(0)$ \\
\hline $\mathrm{LiH}^{\mathrm{c}}$ & ${ }^{3} \Sigma^{+}$ & 6 & $4(3+1)$ & $-7.8997(167)$ & $-7.8939(98)$ & $-7.8940(100)$ & $-7.8854(0)$ \\
\hline $\mathrm{LiH}$ & ${ }^{3} \Sigma^{+}$ & 6 & $4(3+1)$ & $-7.8554(191)$ & $-7.8552(97)$ & $-7.8552(100)$ & $-7.8549(0)$ \\
\hline $\mathrm{BeH}^{+}$ & ${ }^{1} \Sigma^{+}$ & 6 & $4(2+2)$ & $-14.8452(106)$ & $-14.8439(100)$ & $-14.8438(100)$ & $-14.8226(0)$ \\
\hline $\mathrm{BH}^{+}$ & ${ }^{2} \Sigma^{+}$ & 6 & $5(3+2)$ & $-24.8169(151)$ & $-24.8015(100)$ & $-24.8015(100)$ & $-24.7712(0)$ \\
\hline $\mathrm{BH}$ & ${ }^{1} \Sigma^{+}$ & 6 & $6(3+3)$ & $-25.1234(211)$ & $-25.0630(106)$ & $-25.0593(100)$ & $-25.0015(0)$ \\
\hline $\mathrm{CH}^{+}$ & ${ }^{1} \Sigma^{+}$ & 6 & $6(3+3)$ & $-37.9618(227)$ & $-37.8896(107)$ & $-37.8853(100)$ & $-37.8251(0)$ \\
\hline $\mathrm{CH}^{-}$ & ${ }^{3} \Sigma^{-}$ & 6 & $8(5+3)$ & $-37.9834(148)$ & $-37.9714(99)$ & $-37.9718(100)$ & $-37.9477(0)$ \\
\hline $\mathrm{CH}$ & ${ }^{2} \Pi$ & 6 & $7(4+3)$ & $-38.2472(240)$ & $-38.1917(111)$ & $-38.1871(100)$ & $-38.1443(0)$ \\
\hline $\mathrm{NH}^{+}$ & ${ }^{2} \Pi$ & 6 & $7(4+3)$ & $-54.4510(248)$ & $-54.3957(111)$ & $-54.3914(100)$ & $-54.3510(0)$ \\
\hline $\mathrm{NH}^{-}$ & ${ }^{2} \Pi$ & 6 & $9(5+4)$ & $-54.5292(161)$ & $-54.5150(99)$ & $-54.5151(100)$ & $-54.4920(0)$ \\
\hline $\mathrm{NH}$ & ${ }^{3} \Sigma^{-}$ & 6 & $8(5+3)$ & $-54.8280(144)$ & $-54.8160(100)$ & $-54.8161(100)$ & $-54.7887(0)$ \\
\hline $\mathrm{OH}^{+}$ & ${ }^{3} \Sigma^{-}$ & 6 & $8(5+3)$ & $-74.7805(138)$ & $-74.7719(100)$ & $-74.7720(100)$ & $-74.7491(0)$ \\
\hline $\mathrm{OH}^{-}$ & ${ }^{1} \Sigma^{+}$ & 6 & $10(5+5)$ & $-74.8127(100)$ & $-74.8112(95)$ & $-74.8127(100)$ & $-74.7851(0)$ \\
\hline $\mathrm{OH}$ & ${ }^{2} \Pi$ & 6 & $9(5+4)$ & $-75.1164(158)$ & $-75.1013(99)$ & $-75.1014(100)$ & $-75.0756(0)$ \\
\hline $\mathrm{HF}^{+}$ & ${ }^{2} \Pi$ & 6 & $9(5+4)$ & $-99.1376(153)$ & $-99.1278(100)$ & $-99.1279(100)$ & $-99.1096(0)$ \\
\hline $\mathrm{HF}$ & ${ }^{1} \Sigma^{+}$ & 6 & $10(5+5)$ & $-99.5258(100)$ & $-99.5229(89)$ & $-99.5258(100)$ & $-99.4998(0)$ \\
\hline $\mathrm{BH}_{2}$ & ${ }^{2} A_{1}$ & 7 & $7(4+3)$ & $-25.7549(235)$ & $-25.7089(115)$ & $-25.7031(100)$ & $-25.6649(0)$ \\
\hline $\mathrm{BH}_{2}$ & ${ }^{2} B_{1}$ & 7 & $7(4+3)$ & $-25.7317(233)$ & $-25.6837(113)$ & $-25.6783(100)$ & $-25.6383(0)$ \\
\hline $\mathrm{CH}_{2}$ & ${ }^{1} A_{1}$ & 7 & $8(4+4)$ & $-38.9301(294)$ & $-38.8228(119)$ & $-38.8110(100)$ & $-38.7497(0)$ \\
\hline $\mathrm{CH}_{2}$ & ${ }^{3} B_{1}$ & 7 & $8(5+3)$ & $-38.9043(214)$ & $-38.8566(107)$ & $-38.8534(100)$ & $-38.8089(0)$ \\
\hline $\mathrm{CH}_{2}$ & ${ }^{3} \Sigma_{u}^{-}$ & 7 & $8(5+3)$ & $-38.8836(187)$ & $-38.8358(103)$ & $-38.8342(100)$ & $-38.7772(0)$ \\
\hline $\mathrm{NH}_{2}$ & ${ }^{2} A_{1}$ & 7 & $9(5+4)$ & $-55.4134(244)$ & $-55.3570(111)$ & $-55.3525(100)$ & $-55.3101(0)$ \\
\hline $\mathrm{NH}_{2}$ & ${ }^{2} B_{1}$ & 7 & $9(5+4)$ & $-55.4856(243)$ & $-55.4195(108)$ & $-55.4157(100)$ & $-55.3670(0)$ \\
\hline $\mathrm{H}_{2} \mathrm{O}$ & ${ }^{1} A_{1}$ & 7 & $10(5+5)$ & $-75.7953(232)$ & $-75.7310(104)$ & $-75.7290(100)$ & $-75.6789(0)$ \\
\hline $\mathrm{H}_{2} \mathrm{O}^{+}$ & ${ }^{2} A_{1}$ & 7 & $9(5+4)$ & $-75.4912(262)$ & $-75.4218(106)$ & $-75.4192(100)$ & $-75.3748(0)$ \\
\hline $\mathrm{FH}_{2}^{+}$ & ${ }^{1} A_{1}$ & 7 & $10(5+5)$ & $-99.8894(244)$ & $-99.8305(103)$ & $-99.8294(100)$ & $-99.7879(0)$ \\
\hline $\mathrm{BH}_{3}$ & ${ }^{1} A_{1}$ & 8 & $8(4+4)$ & $-26.4681(258)$ & $-26.3932(120)$ & $-26.3827(100)$ & $-26.3287(0)$ \\
\hline $\mathrm{CH}_{3}$ & ${ }^{2} \mathrm{~A}_{2}$ & 8 & $9(5+4)$ & $-39.6375(290)$ & $-39.5283(117)$ & $-39.5178(100)$ & $-39.4547(0)$ \\
\hline $\mathrm{NH}_{3}$ & ${ }^{1} A_{1}$ & 8 & $10(5+5)$ & $-56.2061(334)$ & $-56.0617(115)$ & $-56.0516(100)$ & $-55.9855(0)$ \\
\hline $\mathrm{NH}_{3}$ (dis) & ${ }^{1} A$ & 8 & $10(5+5)$ & $-56.1808(326)$ & $-56.0394(115)$ & $-56.0293(100)$ & $-55.9622(0)$ \\
\hline $\mathrm{H}_{3} \mathrm{O}^{+}$ & ${ }^{1} A_{1}$ & 8 & $10(5+5)$ & $-75.9422(276)$ & $-75.8636(103)$ & $-75.8621(100)$ & $-75.8166(0)$ \\
\hline
\end{tabular}

${ }^{\mathrm{a}}$ Number of active MOs.

${ }^{\mathrm{b}}$ Number of electrons with the number of $\alpha$ and $\beta$ electrons in parentheses.

${ }^{\mathrm{c}}$ Basis set is double- $\zeta$.

${ }^{\mathrm{d}} \mathrm{Basis}$ set is triple- $\zeta$.

by renumbering $i=i_{1}+\left\{\left[i_{2}\left(i_{2}-1\right)\right] / 2\right\}$ if $i_{1}>i_{2}$ and discarding $P$ when $i_{1} \leqslant i_{2}$. This helps to cut down unnecessary variables and to automatically assume that 2-RDM has antisymmetric property. Similarly, the $Q$ matrix and other linear constraints are also folded. Note that the $G$ matrix does not have such a symmetry property, so that we use all the elements.

The present method involves very large number of linear constraints and may not be efficient; a merit is that the SDPA program is used without any modification. However, if we make a problem-specific SDP solver, it would be much more efficient than the present one, and such study is now in progress.

The DMVT formulated above has been applied to the ground states of different space and spin symmetries of neutral and charged species of 16 different atoms and molecules. They are $\operatorname{Be}\left({ }^{1} S\right), \operatorname{Be}\left({ }^{3} S\right), \operatorname{LiH}\left({ }^{1} \Sigma^{+}\right), \operatorname{LiH}\left({ }^{3} \Sigma^{+}\right), \mathrm{BeH}^{+}$
$\mathrm{BH}^{+}, \mathrm{BH}, \mathrm{CH}^{+}, \mathrm{CH}, \mathrm{CH}^{-}, \mathrm{NH}^{+}, \mathrm{NH}^{-}, \mathrm{NH}, \mathrm{OH}^{+}, \mathrm{OH}^{-}$, $\mathrm{OH}, \mathrm{HF}^{+}, \mathrm{HF}, \mathrm{BH}_{2}\left({ }^{2} A_{1}\right), \mathrm{BH}_{2}\left({ }^{2} B_{1}\right), \mathrm{CH}_{2}\left({ }^{1} A_{1}\right), \mathrm{CH}_{2}\left({ }^{3} B_{1}\right)$, linear $\mathrm{CH}_{2}\left({ }^{3} \Sigma_{u}^{-}\right), \mathrm{NH}_{2}\left({ }^{2} A_{1}\right), \mathrm{NH}_{2}\left({ }^{2} B_{1}\right), \mathrm{H}_{2} \mathrm{O}, \mathrm{H}_{2} \mathrm{O}^{+}, \mathrm{FH}_{2}^{+}$, $\mathrm{BH}_{3}, \mathrm{CH}_{3}, \mathrm{NH}_{3}, \mathrm{NH}_{3}$ (dis) ("dis" stands for distorted in the sense that one bond length is shortened by 0.9 time, another one is lengthened by 1.1 time) and $\mathrm{H}_{3} \mathrm{O}^{+}$.

We used three different basis sets, double and triple- $\zeta$ $s$-type GTOs and STO-6G, for Be, and double- $\zeta s$-type GTOs by Huzinaga ${ }^{20}$ and Dunning ${ }^{21}$ and STO-6G for LiH. For all the other molecules, we used the STO-6G basis set. ${ }^{22}$ The geometries we used are the experimental ones. ${ }^{23,24}$

\section{RESULTS AND DISCUSSION}

We show in Table I the total energy of the system calculated by the present method and in parentheses the calculated correlation energy in percentage relative to the 
TABLE II. Dipole moments calulated by the DMVT with $P+Q$ and $P$ $+Q+G$ conditions compared with those obtained by the wave function method. The basis set is STO-6G except for notice.

\begin{tabular}{lccccc}
\hline \hline Molecule & State & $\mathrm{DM}(P+Q)$ & $\mathrm{DM}(P+Q+G)$ & Full CI & Hartree-Fock \\
\hline $\mathrm{LiH}^{\mathrm{a}}$ & ${ }^{1} \Sigma^{+}$ & 1.6445 & 1.6164 & 1.6192 & 2.0764 \\
$\mathrm{LiH}$ & ${ }^{1} \Sigma^{+}$ & 1.7372 & 1.7523 & 1.7519 & 1.9339 \\
$\mathrm{LiH}^{\mathrm{a}}$ & ${ }^{3} \Sigma^{+}$ & 0.6225 & 0.6258 & 0.6258 & 0.6261 \\
$\mathrm{LiH}^{3} \Sigma^{+}$ & 1.5897 & 1.5906 & 1.5907 & 1.5915 \\
$\mathrm{BeH}^{+}$ & ${ }^{1} \Sigma^{+}$ & 1.3203 & 1.3188 & 1.3196 & 1.2987 \\
$\mathrm{BH}^{+}$ & ${ }^{2} \Sigma^{+}$ & 0.0495 & 0.0223 & 0.0223 & 0.0197 \\
$\mathrm{BH}^{1}$ & ${ }^{1}{ }^{+}$ & 0.2833 & 0.2935 & 0.2994 & 0.3806 \\
$\mathrm{CH}^{+}$ & ${ }^{1} \Sigma^{+}$ & 0.6893 & 0.6764 & 0.6905 & 0.7253 \\
$\mathrm{CH}^{-}$ & ${ }^{3} \Sigma$ & 0.1826 & 0.1925 & 0.1929 & 0.1669 \\
$\mathrm{CH}^{2}$ & ${ }^{2} \Pi$ & 0.6016 & 0.4878 & 0.5044 & 0.4406 \\
$\mathrm{NH}^{+}$ & ${ }^{2} \Pi$ & 0.8937 & 0.8729 & 0.8804 & 0.8789 \\
$\mathrm{NH}^{-}$ & ${ }^{2} \Pi$ & 0.1359 & 0.1311 & 0.1321 & 0.1431 \\
$\mathrm{NH}^{3} \mathrm{H}^{-}$ & 0.4730 & 0.4995 & 0.4996 & 0.5233 \\
$\mathrm{OH}^{+}$ & ${ }^{3} \Sigma$ & 0.9988 & 0.9741 & 0.9742 & 0.9875 \\
$\mathrm{OH}^{-}$ & ${ }^{1} \Sigma$ & 0.0620 & 0.0637 & 0.0620 & 0.0725 \\
$\mathrm{OH}^{2}$ & ${ }^{2} \Pi$ & 0.4497 & 0.4738 & 0.4745 & 0.5166 \\
$\mathrm{HF}^{+}$ & ${ }^{2} \Pi$ & 0.9600 & 0.9993 & 0.9999 & 1.0786 \\
$\mathrm{HF}^{1}{ }^{+}{ }^{+}$ & 0.5420 & 0.5383 & 0.5420 & 0.5228 \\
$\mathrm{BH}_{2}$ & ${ }^{2} A_{1}$ & 0.0037 & 0.0328 & 0.0344 & 0.0466 \\
$\mathrm{CH}_{2}$ & ${ }^{1} A_{1}$ & 0.2435 & 0.5057 & 0.5293 & 0.6224 \\
$\mathrm{CH}_{2}$ & ${ }^{3} B_{1}$ & 0.0838 & 0.0857 & 0.0934 & 0.1006 \\
$\mathrm{NH}_{2}$ & ${ }^{2} A_{1}$ & 0.5170 & 0.5407 & 0.5509 & 0.5580 \\
$\mathrm{NH}_{2}$ & ${ }^{2} B_{1}$ & 0.6433 & 0.6816 & 0.6896 & 0.7200 \\
$\mathrm{H}_{2} \mathrm{O}$ & ${ }^{1} A_{1}$ & 0.5993 & 0.6460 & 0.6487 & 0.6927 \\
$\mathrm{H}_{2} \mathrm{O}^{+}$ & ${ }^{2} A_{1}$ & 0.8718 & 0.9857 & 0.9920 & 1.0724 \\
$\mathrm{FH}_{2}^{+}$ & ${ }^{1} A_{1}$ & 1.0368 & 1.0429 & 1.0437 & 1.0560 \\
$\mathrm{NH}_{3}$ & ${ }^{1} A_{1}$ & 0.6903 & 0.6901 & 0.6922 & 0.6935 \\
$\mathrm{NH}_{3}(\mathrm{dis})$ & ${ }^{1} A$ & 0.6660 & 0.6634 & 0.6767 & 0.6937 \\
$\mathrm{H}_{3} \mathrm{O}^{+}$ & ${ }^{1} A_{1}$ & 1.4162 & 1.4286 & 1.4289 & 1.4320 \\
\hline \hline
\end{tabular}

${ }^{\mathrm{a}}$ Basis set is double- $\zeta$.

Hartree-Fock (0\%) and full-CI (100\%) results. Two types of SDP relaxation calculations are performed. One uses the $P$ and $Q$ conditions together with the seven conditions given by Eqs. (2.3)-(2.10); it is referred to as $\operatorname{DM}(P+Q)$. The other uses the $G$ condition additionally and it is denoted as $\mathrm{DM}(P+Q+G)$.

We first examine the results of $\operatorname{DM}(P+Q)$ calculations. We see that the results for $\mathrm{OH}^{-}$and $\mathrm{HF}$ are excellent, but this is not a good news but simply due to the too restrictive variational space: 10 electrons are distributed into six orbitals and therefore in this case $P+Q$ condition gives the complete $N$-representability condition (2 hole system). ${ }^{25}$ Similarly, the extent of overshooting is relatively small because the variational space is too restrictive. When the variation is reasonably free, the $\mathrm{DM}(P+Q)$ energy overshoots too much the full-CI energy up to $334 \%$ of the full-CI correlation energy for $\mathrm{NH}_{3}$. This result shows that the $P+Q$ condition together with the above seven conditions is still too far from the complete $N$-representability condition.

When we impose further the $G$ condition, we obtain the results shown under $\operatorname{DM}(P+Q+G)$. They are much improved in comparison with the results of $\operatorname{DM}(P+Q)$. The calculated correlation energy percentages range within $100 \%-110 \%$ for atoms and diatomic molecules, while they range in $110 \%-120 \%$ for triatomic molecules. This means that the $G$ condition is a nice restrictive condition for the
TABLE III. Virial coefficients calulated by the DMVT with the $P+Q$ and $P+Q+G$ conditions compared with those obtained by the wave function methods. The basis set is STO-6G except for notice.

\begin{tabular}{|c|c|c|c|c|c|}
\hline System & State & $\operatorname{DM}(P+Q)$ & $\mathrm{DM}(P+Q+G)$ & Full CI & Hartree-Fock \\
\hline $\mathrm{Be}^{\mathrm{a}}$ & ${ }^{1} S$ & 1.9975 & 1.9989 & 1.9989 & 1.9994 \\
\hline $\mathrm{Be}$ & ${ }^{1} S$ & 1.9621 & 1.9614 & 1.9614 & 1.9558 \\
\hline $\mathrm{Be}^{\mathrm{b}}$ & ${ }^{1} S$ & 2.0017 & 2.0006 & 2.0006 & 2.0000 \\
\hline $\mathrm{Be}^{\mathrm{a}}$ & ${ }^{3} S$ & 1.7459 & 1.7461 & 1.7461 & 1.7464 \\
\hline $\mathrm{Be}^{\mathrm{b}}$ & ${ }^{3} S$ & 1.9774 & 1.9766 & 1.9766 & 1.9759 \\
\hline $\mathrm{LiH}^{\mathrm{a}}$ & ${ }^{1} \Sigma^{+}$ & 2.0038 & 1.9977 & 1.9977 & 1.9929 \\
\hline $\mathrm{LiH}$ & ${ }^{1} \Sigma^{+}$ & 1.9832 & 1.9826 & 1.9826 & 1.9837 \\
\hline $\mathrm{LiH}^{\mathrm{a}}$ & ${ }^{3} \Sigma^{+}$ & 1.9908 & 1.9875 & 1.9875 & 1.9826 \\
\hline $\mathrm{LiH}$ & ${ }^{3} \Sigma^{+}$ & 1.9579 & 1.9577 & 1.9577 & 1.9574 \\
\hline $\mathrm{BeH}^{+}$ & ${ }^{1} \Sigma^{+}$ & 2.0036 & 2.0031 & 2.0031 & 2.0041 \\
\hline $\mathrm{BH}^{+}$ & ${ }^{2} \Sigma^{+}$ & 1.9918 & 1.9919 & 1.9918 & 1.9931 \\
\hline $\mathrm{BH}$ & ${ }^{1} \Sigma^{+}$ & 1.9574 & 1.9565 & 1.9566 & 1.9550 \\
\hline $\mathrm{CH}^{+}$ & ${ }^{1} \Sigma^{+}$ & 2.0044 & 2.0040 & 2.0039 & 2.0025 \\
\hline $\mathrm{CH}^{-}$ & ${ }^{3} \Sigma^{-}$ & 1.9393 & 1.9392 & 1.9393 & 1.9396 \\
\hline $\mathrm{CH}$ & ${ }^{2} \Pi$ & 1.9781 & 1.9778 & 1.9777 & 1.9773 \\
\hline $\mathrm{NH}^{+}$ & ${ }^{2} \Pi$ & 2.0164 & 2.0156 & 2.0154 & 2.0144 \\
\hline $\mathrm{NH}^{-}$ & ${ }^{2} \Pi$ & 1.9596 & 1.9597 & 1.9597 & 1.9601 \\
\hline $\mathrm{NH}$ & ${ }^{3} \Sigma^{-}$ & 1.9941 & 1.9939 & 1.9938 & 1.9939 \\
\hline $\mathrm{OH}^{+}$ & ${ }^{3} \Sigma^{-}$ & 2.0199 & 2.0194 & 2.0194 & 2.0183 \\
\hline $\mathrm{OH}^{-}$ & ${ }^{1} \Sigma^{+}$ & 1.9672 & 1.9671 & 1.9672 & 1.9678 \\
\hline $\mathrm{OH}$ & ${ }^{2} \Pi$ & 1.9967 & 1.9965 & 1.9965 & 1.9965 \\
\hline $\mathrm{HF}^{+}$ & ${ }^{2} \Pi$ & 2.0218 & 2.0212 & 2.0212 & 2.0201 \\
\hline $\mathrm{HF}$ & ${ }^{1} \Sigma^{+}$ & 2.0001 & 2.0001 & 2.0001 & 1.9999 \\
\hline $\mathrm{BH}_{2}$ & ${ }^{2} A_{1}$ & 1.9722 & 1.9727 & 1.9731 & 1.9738 \\
\hline $\mathrm{BH}_{2}$ & ${ }^{2} B_{1}$ & 1.9699 & 1.9702 & 1.9705 & 1.9712 \\
\hline $\mathrm{CH}_{2}$ & ${ }^{1} A_{1}$ & 1.9849 & 1.9840 & 1.9840 & 1.9841 \\
\hline $\mathrm{CH}_{2}$ & ${ }^{3} B_{1}$ & 1.9889 & 1.9884 & 1.9886 & 1.9886 \\
\hline $\mathrm{CH}_{2}$ & ${ }^{3} \Sigma_{u}^{-}$ & 1.9882 & 1.9876 & 1.9878 & 1.9877 \\
\hline $\mathrm{NH}_{2}$ & ${ }^{2} A_{1}$ & 1.9950 & 1.9943 & 1.9942 & 1.9940 \\
\hline $\mathrm{NH}_{2}$ & ${ }^{2} B_{1}$ & 1.9956 & 1.9955 & 1.9955 & 1.9956 \\
\hline $\mathrm{H}_{2} \mathrm{O}$ & ${ }^{1} A_{1}$ & 1.9966 & 1.9968 & 1.9968 & 1.9967 \\
\hline $\mathrm{H}_{2} \mathrm{O}^{+}$ & ${ }^{2} A_{1}$ & 2.0176 & 2.0152 & 2.0151 & 2.0138 \\
\hline $\mathrm{FH}_{2}^{+}$ & ${ }^{1} A_{1}$ & 2.0183 & 2.0159 & 2.0158 & 2.0143 \\
\hline $\mathrm{BH}_{3}$ & ${ }^{1} A_{1}$ & 1.9835 & 1.9836 & 1.9843 & 1.9853 \\
\hline $\mathrm{CH}_{3}$ & ${ }^{2} A_{2}$ & 1.9941 & 1.9939 & 1.9944 & 1.9948 \\
\hline $\mathrm{NH}_{3}$ & ${ }^{1} A_{1}$ & 1.9981 & 1.9985 & 1.9984 & 1.9985 \\
\hline $\mathrm{NH}_{3}$ (dis) & ${ }^{1} A$ & 1.9973 & 1.9976 & 1.9974 & 1.9976 \\
\hline $\mathrm{H}_{3} \mathrm{O}^{+}$ & ${ }^{1} A_{1}$ & 1.9972 & 1.9941 & 1.9941 & 1.9934 \\
\hline
\end{tabular}

${ }^{a}$ Basis set is double- $\zeta$.

${ }^{\mathrm{b}} \mathrm{Basis}$ set is triple- $\zeta$.

$N$-representability. We investigated distorted ammonia to examine whether the spatial symmetry affects the $N$-representability condition, however, this calculation shows that there is no effect by such a small distortion; the accuracies of the two calculations are almost the same.

The SDP variational method should give, in principle, a lower bound for energy, however, compared to the full-CI results, the breakdown where the calculated SDPA energy is higher than the full-CI energy occurs for $\mathrm{LiH}\left({ }^{3} \Sigma\right.$, STO-6G), $\mathrm{LiH}\left({ }^{3} \Sigma\right.$, double- $\left.\zeta\right), \mathrm{CH}^{-}, \mathrm{NH}^{-}, \mathrm{OH}, \mathrm{OH}^{-}$, and $\mathrm{HF}$, though the violations are within 1 mhartree. It seems that these breakdowns are related to the numerical errors in the SDPA procedure, which we discuss later.

In Table II, we show the (nonzero) dipole moments of the molecules calculated here. The dipole moment obtained at the level of $\mathrm{DM}(P+Q)$ is not so good. In particular, those for $\mathrm{CH}, \mathrm{NH}^{+}, \mathrm{CH}_{2}, \mathrm{H}_{2} \mathrm{O}$, and $\mathrm{H}_{2} \mathrm{O}^{+}$are worse than the Hartree-Fock results. At the $\operatorname{DM}(P+Q+G)$ level, how- 
TABLE IV. Number of the constraints and the numerical errors of the $\operatorname{DM}(P+Q)$ calculations. The basis set is STO-6G except for notice.

\begin{tabular}{|c|c|c|c|c|c|c|c|}
\hline System & State & $\begin{array}{l}\text { Active }^{\mathrm{a}} \\
\text { MOs }\end{array}$ & $\begin{array}{c}\operatorname{Ele}(\alpha+\beta)^{\mathrm{b}} \\
\text { constraints }\end{array}$ & $\begin{array}{c}\text { No. of } \\
\text { constraints }\end{array}$ & $\begin{array}{c}\text { Primal feasible } \\
\text { error }\end{array}$ & $\begin{array}{c}\text { Dual feasible } \\
\text { error }\end{array}$ & Gap \\
\hline $\mathrm{Be}^{\mathrm{c}}$ & ${ }^{1} S$ & 4 & $4(2+2)$ & 183 & $1.87 \times 10^{-14}$ & $9.24 \times 10^{-11}$ & $1.17 \times 10^{-9}$ \\
\hline $\mathrm{Be}$ & ${ }^{1} S$ & 5 & $4(2+2)$ & 440 & $7.87 \times 10^{-14}$ & $4.35 \times 10^{-11}$ & $8.03 \times 10^{-13}$ \\
\hline $\mathrm{Be}^{\mathrm{d}}$ & ${ }^{1} S$ & 5 & $4(2+2)$ & 440 & $5.49 \times 10^{-14}$ & $1.41 \times 10^{-10}$ & $1.89 \times 10^{-11}$ \\
\hline $\mathrm{Be}^{\mathrm{c}}$ & ${ }^{3} S$ & 4 & $4(3+1)$ & 183 & $4.12 \times 10^{-11}$ & $1.17 \times 10^{-7}$ & $3.87 \times 10^{-8}$ \\
\hline $\mathrm{Be}^{\mathrm{d}}$ & ${ }^{3} S$ & 5 & $4(3+1)$ & 440 & $7.86 \times 10^{-12}$ & $1.12 \times 10^{-7}$ & $9.58 \times 10^{-9}$ \\
\hline $\mathrm{LiH}^{\mathrm{c}}$ & ${ }^{1} \Sigma^{+}$ & 6 & $4(2+2)$ & 911 & $7.24 \times 10^{-14}$ & $9.24 \times 10^{-9}$ & $3.46 \times 10^{-9}$ \\
\hline $\mathrm{LiH}$ & ${ }^{1} \Sigma^{+}$ & 6 & $4(2+2)$ & 911 & $8.52 \times 10^{-14}$ & $1.08 \times 10^{-9}$ & $4.76 \times 10^{-11}$ \\
\hline $\mathrm{LiH}^{\mathrm{c}}$ & ${ }^{3} \Sigma^{+}$ & 6 & $4(3+1)$ & 911 & $3.80 \times 10^{-12}$ & $1.75 \times 10^{-6}$ & $3.64 \times 10^{-7}$ \\
\hline $\mathrm{LiH}$ & ${ }^{3} \Sigma^{+}$ & 6 & $4(3+1)$ & 911 & $3.41 \times 10^{-11}$ & $4.57 \times 10^{-8}$ & $1.89 \times 10^{-8}$ \\
\hline $\mathrm{BeH}^{+}$ & ${ }^{1} \Sigma^{+}$ & 6 & $4(2+2)$ & 911 & $1.16 \times 10^{-13}$ & $3.02 \times 10^{-11}$ & $5.73 \times 10^{-13}$ \\
\hline $\mathrm{BH}^{+}$ & ${ }^{2} \Sigma^{+}$ & 6 & $5(3+2)$ & 911 & $6.69 \times 10^{-14}$ & $1.86 \times 10^{-10}$ & $3.26 \times 10^{-12}$ \\
\hline $\mathrm{BH}$ & ${ }^{1} \Sigma^{+}$ & 6 & $6(3+3)$ & 911 & $4.43 \times 10^{-14}$ & $8.29 \times 10^{-10}$ & $2.41 \times 10^{-11}$ \\
\hline $\mathrm{CH}^{+}$ & ${ }^{1} \Sigma^{+}$ & 6 & $6(3+3)$ & 911 & $3.62 \times 10^{-14}$ & $1.30 \times 10^{-9}$ & $9.62 \times 10^{-12}$ \\
\hline $\mathrm{CH}^{-}$ & ${ }^{3} \Sigma^{-}$ & 6 & $8(5+3)$ & 911 & $5.49 \times 10^{-12}$ & $8.97 \times 10^{-8}$ & $6.05 \times 10^{-9}$ \\
\hline $\mathrm{CH}$ & ${ }^{2} \Pi$ & 6 & $7(4+3)$ & 911 & $5.91 \times 10^{-14}$ & $2.23 \times 10^{-9}$ & $2.80 \times 10^{-11}$ \\
\hline $\mathrm{NH}^{+}$ & ${ }^{2} \Pi$ & 6 & $7(4+3)$ & 911 & $3.15 \times 10^{-14}$ & $9.98 \times 10^{-10}$ & $1.49 \times 10^{-11}$ \\
\hline $\mathrm{NH}^{-}$ & ${ }^{2} \Pi$ & 6 & $9(5+4)$ & 911 & $8.57 \times 10^{-12}$ & $7.06 \times 10^{-8}$ & $3.70 \times 10^{-9}$ \\
\hline NH & ${ }^{3} \Sigma^{-}$ & 6 & $8(5+3)$ & 911 & $9.79 \times 10^{-12}$ & $4.97 \times 10^{-7}$ & $1.85 \times 10^{-8}$ \\
\hline $\mathrm{OH}^{+}$ & ${ }^{3} \Sigma^{-}$ & 6 & $8(5+3)$ & 911 & $6.77 \times 10^{-12}$ & $8.16 \times 10^{-8}$ & $2.49 \times 10^{-9}$ \\
\hline $\mathrm{OH}^{-}$ & ${ }^{1} \Sigma^{+}$ & 6 & $10(5+5)$ & 911 & $7.50 \times 10^{-12}$ & $5.25 \times 10^{-7}$ & $7.32 \times 10^{-8}$ \\
\hline $\mathrm{OH}$ & ${ }^{2} \Pi$ & 6 & $9(5+4)$ & 911 & $5.54 \times 10^{-12}$ & $1.35 \times 10^{-7}$ & $3.48 \times 10^{-9}$ \\
\hline $\mathrm{HF}^{+}$ & ${ }^{2} \Pi$ & 6 & $9(5+4)$ & 911 & $2.36 \times 10^{-12}$ & $8.42 \times 10^{-8}$ & $2.96 \times 10^{-9}$ \\
\hline $\mathrm{HF}$ & ${ }^{1} \Sigma^{+}$ & 6 & $10(5+5)$ & 911 & $6.82 \times 10^{-12}$ & $1.12 \times 10^{-7}$ & $6.65 \times 10^{-9}$ \\
\hline $\mathrm{BH}_{2}$ & ${ }^{2} A_{1}$ & 7 & $7(4+3)$ & 1692 & $6.43 \times 10^{-14}$ & $4.94 \times 10^{-10}$ & $4.65 \times 10^{-11}$ \\
\hline $\mathrm{BH}_{2}$ & ${ }^{2} B_{1}$ & 7 & $7(4+3)$ & 1692 & $5.66 \times 10^{-14}$ & $8.16 \times 10^{-12}$ & $3.20 \times 10^{-12}$ \\
\hline $\mathrm{CH}_{2}$ & ${ }^{1} A_{1}$ & 7 & $8(4+4)$ & 1692 & $3.25 \times 10^{-14}$ & $3.26 \times 10^{-11}$ & $3.19 \times 10^{-10}$ \\
\hline $\mathrm{CH}_{2}$ & ${ }^{3} B_{1}$ & 7 & $8(5+3)$ & 1692 & $3.98 \times 10^{-14}$ & $5.94 \times 10^{-11}$ & $5.75 \times 10^{-10}$ \\
\hline $\mathrm{CH}_{2}$ & ${ }^{3} \Sigma_{u}^{-}$ & 7 & $8(5+3)$ & 1692 & $4.12 \times 10^{-14}$ & $6.59 \times 10^{-11}$ & $5.88 \times 10^{-11}$ \\
\hline $\mathrm{NH}_{2}$ & ${ }^{2} A_{1}$ & 7 & $9(5+4)$ & 1692 & $3.63 \times 10^{-14}$ & $1.21 \times 10^{-9}$ & $1.22 \times 10^{-10}$ \\
\hline $\mathrm{NH}_{2}$ & ${ }^{2} B_{1}$ & 7 & $9(5+4)$ & 1692 & $3.99 \times 10^{-14}$ & $2.46 \times 10^{-11}$ & $3.91 \times 10^{-10}$ \\
\hline $\mathrm{H}_{2} \mathrm{O}$ & ${ }^{1} A_{1}$ & 7 & $10(5+5)$ & 1692 & $3.33 \times 10^{-14}$ & $2.88 \times 10^{-10}$ & $1.29 \times 10^{-10}$ \\
\hline $\mathrm{H}_{2} \mathrm{O}^{+}$ & ${ }^{2} A_{1}$ & 7 & $9(5+4)$ & 1692 & $3.31 \times 10^{-14}$ & $3.02 \times 10^{-11}$ & $2.64 \times 10^{-10}$ \\
\hline $\mathrm{FH}_{2}^{+}$ & ${ }^{1} A_{1}$ & 7 & $10(5+5)$ & 1692 & $3.46 \times 10^{-14}$ & $3.19 \times 10^{-10}$ & $1.12 \times 10^{-10}$ \\
\hline $\mathrm{BH}_{3}$ & ${ }^{1} A_{1}$ & 8 & $8(4+4)$ & 2897 & $6.57 \times 10^{-14}$ & $7.62 \times 10^{-10}$ & $3.37 \times 10^{-11}$ \\
\hline $\mathrm{CH}_{3}$ & ${ }^{2} A_{2}$ & 8 & $9(5+4)$ & 2897 & $3.87 \times 10^{-14}$ & $1.12 \times 10^{-11}$ & $8.68 \times 10^{-10}$ \\
\hline $\mathrm{NH}_{3}$ & ${ }^{1} A_{1}$ & 8 & $10(5+5)$ & 2897 & $4.07 \times 10^{-14}$ & $5.16 \times 10^{-11}$ & $1.02 \times 10^{-10}$ \\
\hline $\mathrm{NH}_{3}$ (dis) & ${ }^{1} A$ & 8 & $10(5+5)$ & 2897 & $4.38 \times 10^{-14}$ & $5.87 \times 10^{-10}$ & $1.62 \times 10^{-10}$ \\
\hline $\mathrm{H}_{3} \mathrm{O}^{+}$ & ${ }^{1} A_{1}$ & 8 & $10(5+5)$ & 2897 & $4.13 \times 10^{-14}$ & $5.29 \times 10^{-10}$ & $6.77 \times 10^{-11}$ \\
\hline
\end{tabular}

${ }^{a}$ Number of active MOs.

${ }^{\mathrm{b}}$ Number of electrons with the number of $\alpha$ and $\beta$ electrons in parentheses.

${ }^{\mathrm{c}} \mathrm{Basis}$ set is double- $\zeta$.

${ }^{\mathrm{d}}$ Basis set is triple- $\zeta$.

ever, the dipole moments are drastically improved and all the results well reproduce the full-CI ones, except for $\mathrm{NH}_{3}$ and $\mathrm{NH}_{3}$ (dis) for which even Hartree-Fock calculations give good results and the deviations are very small.

In Table III, we show the virial coefficient $\langle V\rangle /\langle T\rangle$, where $\langle V\rangle$ and $\langle T\rangle$ denote average potential and kinetic energies, respectively, which must be two for completely variational wave function. When we use $\operatorname{DM}(P+Q+G)$ approximation, the calculated virial is almost completely identical with the full-CI result.

Next, we discuss the numerical accuracy of the SDP method. In Tables IV and V, we summarize the number of the constraints and the numerical errors of the $\operatorname{DM}(P+Q)$ and $\mathrm{DM}(P+Q+G)$ calculations. The primal feasible error is defined by

$$
\max \left\{\left|\left[\mathbf{X}-\sum_{i=1}^{m} \mathbf{F}_{i} x_{i}+\mathbf{F}_{0}\right]_{p q}\right|: p, q=1,2, \ldots, n\right\}
$$

and the dual feasible error is defined by

$$
\max \left\{\left|\mathbf{F}_{i} \cdot \mathbf{Y}-c_{i}\right|: i=1,2, \ldots, m\right\} .
$$

The gap denotes the difference between the primal and dual functions defined by

$$
\left|\sum_{i=1}^{m} c_{i} x_{i}-\mathbf{F}_{0} \cdot \mathbf{Y}\right|
$$

These three quantities give criteria of the accuracy of the SDPA. In the SDPA, our object is the minimization of the 
TABLE V. Number of the constraints and the numerical errors of the $\operatorname{DM}(P+Q+G)$ calculations. The basis set is STO-6G except for notice.

\begin{tabular}{|c|c|c|c|c|c|c|c|}
\hline System & State & $\begin{array}{l}\text { Active }^{\mathrm{a}} \\
\text { MOs }\end{array}$ & $\operatorname{Ele}(\alpha+\beta)^{\mathrm{b}}$ & $\begin{array}{c}\text { No. of } \\
\text { constraints }\end{array}$ & $\begin{array}{c}\text { Primal feasible } \\
\text { error }\end{array}$ & $\begin{array}{c}\text { Dual feasible } \\
\text { error }\end{array}$ & Gap \\
\hline $\mathrm{Be}^{\mathrm{c}}$ & ${ }^{1} S$ & 4 & $4(2+2)$ & 983 & $6.55 \times 10^{-11}$ & $1.87 \times 10^{-7}$ & $2.87 \times 10^{-6}$ \\
\hline $\mathrm{Be}$ & ${ }^{1} S$ & 5 & $4(2+2)$ & 2365 & $7.02 \times 10^{-12}$ & $4.93 \times 10^{-7}$ & $1.42 \times 10^{-6}$ \\
\hline $\mathrm{Be}^{\mathrm{d}}$ & ${ }^{1} S$ & 5 & $4(2+2)$ & 2365 & $7.92 \times 10^{-11}$ & $4.15 \times 10^{-7}$ & $2.48 \times 10^{-6}$ \\
\hline $\mathrm{Be}^{\mathrm{c}}$ & ${ }^{3} S$ & 4 & $4(3+1)$ & 983 & $1.12 \times 10^{-10}$ & $7.44 \times 10^{-7}$ & $3.08 \times 10^{-6}$ \\
\hline $\mathrm{Be}^{\mathrm{d}}$ & ${ }^{3} S$ & 5 & $4(3+1)$ & 2365 & $1.41 \times 10^{-11}$ & $1.65 \times 10^{-7}$ & $2.94 \times 10^{-7}$ \\
\hline $\mathrm{LiH}^{\mathrm{c}}$ & ${ }^{1} \Sigma^{+}$ & 6 & $4(2+2)$ & 4871 & $5.72 \times 10^{-12}$ & $2.33 \times 10^{-5}$ & $4.50 \times 10^{-6}$ \\
\hline $\mathrm{LiH}$ & ${ }^{1} \Sigma^{+}$ & 6 & $4(2+2)$ & 4871 & $5.77 \times 10^{-12}$ & $7.55 \times 10^{-8}$ & $1.69 \times 10^{-6}$ \\
\hline $\mathrm{LiH}^{\mathrm{c}}$ & ${ }^{3} \Sigma^{+}$ & 6 & $4(3+1)$ & 4871 & $3.53 \times 10^{-11}$ & $6.35 \times 10^{-7}$ & $2.41 \times 10^{-5}$ \\
\hline $\mathrm{LiH}$ & ${ }^{3} \Sigma^{+}$ & 6 & $4(3+1)$ & 4871 & $5.79 \times 10^{-11}$ & $6.56 \times 10^{-7}$ & $2.58 \times 10^{-6}$ \\
\hline $\mathrm{BeH}^{+}$ & ${ }^{1} \Sigma^{+}$ & 6 & $4(2+2)$ & 4871 & $2.42 \times 10^{-11}$ & $1.88 \times 10^{-7}$ & $1.93 \times 10^{-6}$ \\
\hline $\mathrm{BH}^{+}$ & ${ }^{2} \Sigma^{+}$ & 6 & $5(3+2)$ & 4871 & $7.84 \times 10^{-12}$ & $7.50 \times 10^{-8}$ & $7.82 \times 10^{-7}$ \\
\hline $\mathrm{BH}$ & ${ }^{1} \Sigma^{+}$ & 6 & $6(3+3)$ & 4871 & $2.80 \times 10^{-11}$ & $1.43 \times 10^{-5}$ & $1.61 \times 10^{-8}$ \\
\hline $\mathrm{CH}^{+}$ & ${ }^{1} \Sigma^{+}$ & 6 & $6(3+3)$ & 4871 & $3.75 \times 10^{-12}$ & $2.41 \times 10^{-7}$ & $9.68 \times 10^{-7}$ \\
\hline $\mathrm{CH}^{-}$ & ${ }^{3} \Sigma^{-}$ & 6 & $8(5+3)$ & 4871 & $1.43 \times 10^{-11}$ & $3.73 \times 10^{-7}$ & $2.13 \times 10^{-5}$ \\
\hline $\mathrm{CH}$ & ${ }^{2} \Pi$ & 6 & $7(4+3)$ & 4871 & $6.06 \times 10^{-12}$ & $8.54 \times 10^{-6}$ & $1.91 \times 10^{-7}$ \\
\hline $\mathrm{NH}^{+}$ & ${ }^{2} \Pi$ & 6 & $7(4+3)$ & 4871 & $4.15 \times 10^{-12}$ & $3.64 \times 10^{-6}$ & $4.98 \times 10^{-7}$ \\
\hline $\mathrm{NH}^{-}$ & ${ }^{2} \Pi$ & 6 & $9(5+4)$ & 4871 & $6.89 \times 10^{-12}$ & $3.39 \times 10^{-6}$ & $1.37 \times 10^{-5}$ \\
\hline $\mathrm{NH}$ & ${ }^{3} \Sigma^{-}$ & 6 & $8(5+3)$ & 4871 & $8.69 \times 10^{-11}$ & $1.88 \times 10^{-5}$ & $2.90 \times 10^{-6}$ \\
\hline $\mathrm{OH}^{+}$ & ${ }^{3} \Sigma^{-}$ & 6 & $8(5+3)$ & 4871 & $7.98 \times 10^{-12}$ & $4.05 \times 10^{-7}$ & $1.90 \times 10^{-6}$ \\
\hline $\mathrm{OH}^{-}$ & ${ }^{1} \Sigma^{+}$ & 6 & $10(5+5)$ & 4871 & $2.54 \times 10^{-11}$ & $5.65 \times 10^{-6}$ & $2.72 \times 10^{-5}$ \\
\hline $\mathrm{OH}$ & ${ }^{2} \Pi$ & 6 & $9(5+4)$ & 4871 & $1.01 \times 10^{-11}$ & $2.78 \times 10^{-6}$ & $6.15 \times 10^{-6}$ \\
\hline $\mathrm{HF}^{+}$ & ${ }^{2} \Pi$ & 6 & $9(5+4)$ & 4871 & $1.09 \times 10^{-11}$ & $1.40 \times 10^{-6}$ & $6.07 \times 10^{-6}$ \\
\hline $\mathrm{HF}$ & ${ }^{1} \Sigma^{+}$ & 6 & $10(5+5)$ & 4871 & $1.39 \times 10^{-11}$ & $7.37 \times 10^{-6}$ & $3.99 \times 10^{-5}$ \\
\hline $\mathrm{BH}_{2}$ & ${ }^{2} A_{1}$ & 7 & $7(4+3)$ & 8993 & $2.50 \times 10^{-12}$ & $8.84 \times 10^{-8}$ & $8.00 \times 10^{-7}$ \\
\hline $\mathrm{BH}_{2}$ & ${ }^{2} B_{1}$ & 7 & $7(4+3)$ & 8993 & $9.48 \times 10^{-12}$ & $1.23 \times 10^{-6}$ & $1.59 \times 10^{-6}$ \\
\hline $\mathrm{CH}_{2}$ & ${ }^{1} A_{1}$ & 7 & $8(4+4)$ & 8993 & $4.14 \times 10^{-12}$ & $3.54 \times 10^{-6}$ & $2.69 \times 10^{-7}$ \\
\hline $\mathrm{CH}_{2}$ & ${ }^{3} B_{1}$ & 7 & $8(5+3)$ & 8993 & $1.57 \times 10^{-10}$ & $4.05 \times 10^{-5}$ & $1.28 \times 10^{-7}$ \\
\hline $\mathrm{CH}_{2}$ & ${ }^{3} \Sigma_{u}^{-}$ & 7 & $8(5+3)$ & 8993 & $4.72 \times 10^{-12}$ & $3.98 \times 10^{-7}$ & $1.58 \times 10^{-6}$ \\
\hline $\mathrm{NH}_{2}$ & ${ }^{2} A_{1}$ & 7 & $9(5+4)$ & 8993 & $7.65 \times 10^{-12}$ & $4.30 \times 10^{-6}$ & $1.30 \times 10^{-6}$ \\
\hline $\mathrm{NH}_{2}$ & ${ }^{2} B_{1}$ & 7 & $9(5+4)$ & 8993 & $2.36 \times 10^{-12}$ & $3.22 \times 10^{-8}$ & $2.16 \times 10^{-6}$ \\
\hline $\mathrm{H}_{2} \mathrm{O}$ & ${ }^{1} A_{1}$ & 7 & $10(5+5)$ & 8993 & $1.54 \times 10^{-12}$ & $1.05 \times 10^{-7}$ & $3.63 \times 10^{-7}$ \\
\hline $\mathrm{H}_{2} \mathrm{O}^{+}$ & ${ }^{2} A_{1}$ & 7 & $9(5+4)$ & 8993 & $1.12 \times 10^{-11}$ & $6.86 \times 10^{-6}$ & $7.63 \times 10^{-7}$ \\
\hline $\mathrm{FH}_{2}^{+}$ & ${ }^{1} A_{1}$ & 7 & $10(5+5)$ & 8993 & $3.78 \times 10^{-12}$ & $5.14 \times 10^{-7}$ & $6.07 \times 10^{-7}$ \\
\hline $\mathrm{BH}_{3}$ & ${ }^{1} A_{1}$ & 8 & $8(4+4)$ & 15313 & $5.02 \times 10^{-11}$ & $5.34 \times 10^{-7}$ & $2.88 \times 10^{-7}$ \\
\hline $\mathrm{CH}_{3}$ & ${ }^{2} A_{2}$ & 8 & $9(5+4)$ & 15313 & $4.26 \times 10^{-12}$ & $6.16 \times 10^{-7}$ & $9.01 \times 10^{-8}$ \\
\hline $\mathrm{NH}_{3}$ & ${ }^{1} A_{1}$ & 8 & $10(5+5)$ & 15313 & $1.65 \times 10^{-12}$ & $4.62 \times 10^{-7}$ & $4.39 \times 10^{-7}$ \\
\hline $\mathrm{NH}_{3}$ (dis) & ${ }^{1} A$ & 8 & $10(5+5)$ & 15313 & $3.29 \times 10^{-12}$ & $1.42 \times 10^{-6}$ & $4.14 \times 10^{-6}$ \\
\hline $\mathrm{H}_{3} \mathrm{O}^{+}$ & ${ }^{1} A_{1}$ & 8 & $10(5+5)$ & 15313 & $1.59 \times 10^{-12}$ & $2.30 \times 10^{-7}$ & $2.24 \times 10^{-6}$ \\
\hline
\end{tabular}

${ }^{a}$ Number of active MOs.

${ }^{b}$ Number of electrons with the number of $\alpha$ and $\beta$ electrons in parentheses.

${ }^{\mathrm{c}} \mathrm{Basis}$ set is double- $\zeta$.

${ }^{\mathrm{d}}$ Basis set is triple- $\zeta$.

dual form of the problem, so that the dual feasible error is an important quantity, indicating the numerical accuracy of the calculation.

For $\mathrm{DM}(P+Q)$, the dual feasible error is in the range of $10^{-7}-10^{-12}$, while for $\operatorname{DM}(P+Q+G)$, it ranges $10^{-5}-10^{-8}$. As the number of the constraints increases drastically in the $P+Q+G$ calculations, the numerical accuracy becomes much worse in the $\operatorname{DM}(P+Q+G)$ results. The gap value shows the same tendency. The worst five are $\mathrm{HF}\left(3.99 \times 10^{-5}\right), \mathrm{OH}^{-}\left(2.72 \times 10^{-5}\right), \mathrm{LiH}\left(\right.$ double- $\zeta,{ }^{3} \Sigma$; $\left.2.41 \times 10^{-5}\right), \mathrm{CH}^{-}\left(2.31 \times 10^{-5}\right)$, and $\mathrm{NH}^{-}\left(1.37 \times 10^{-5}\right)$. We notice that they have the $\operatorname{DM}(P+Q+G)$ energies higher than the full-CI ones, though these values must be lower than the full-CI values. There seems to be some relation between the gap value and the numerical accuracy in the SDPA technique. Another reason is certainly the too small variational freedom in the calculations of $\mathrm{HF}$ and $\mathrm{OH}^{-}$; actually in these cases $P$ and $Q$ conditions are already sufficient; the number of holes is 2 , so the 2-hole system with $Q$ condition is just like performing variational calculation for the 2-electron system with the $P$ condition. Therefore enforcing $P, Q$, and $G$ conditions is essentially the same as enforcing $P$ and $Q$ conditions.

The primal feasible values are very small $\left(10^{-12}-10^{-14}\right)$ for $\operatorname{DM}(P+Q)$ calculations and also small $\left(10^{-10}-10^{-12}\right)$ for $\operatorname{DM}(P+Q+G)$ calculations. We do not find any relationship between the accuracies of the present calculations and the primal feasible errors. So the accuracy of the present calculation seems to be related only to that of the primal problem.

In Table VI, we show the occupation numbers (eigenval- 
TABLE VI. Occupation number calculated by the DMVT with $P+Q$ and $P+Q+G$ conditions compared with those obatined by the wave function methods, full $\mathrm{CI}$, and Hartree-Fock, for $\mathrm{Be}, \mathrm{H}_{2} \mathrm{O}$, and $\mathrm{CH}_{2}$.

\begin{tabular}{|c|c|c|c|c|}
\hline System, state, basis & $\mathrm{DM}(P+Q)$ & $\mathrm{DM}(P+Q+G)$ & Full CI & Hartree-Fock \\
\hline \multirow[t]{3}{*}{ Be, ${ }^{1} S$, STO-6G } & $0.036641 \times 4,0.036642 \times 2$ & $0.035909 \times 6$ & $0.035901 \times 6$ & $0 \times 6$ \\
\hline & $0.890243 \times 2$ & $0.892275 \times 2$ & $0.892298 \times 2$ & $1 \times 4$ \\
\hline & $0.999832 \times 2$ & $0.999997 \times 2$ & $0.999998 \times 2$ & \\
\hline \multirow[t]{5}{*}{ Be, ${ }^{1} S$, triple- $\zeta$} & $0.000202 \times 2$ & $0.000064 \times 2$ & $0.000055 \times 2$ & $0 \times 6$ \\
\hline & $0.001041 \times 2$ & $0.000595 \times 2$ & $0.000590 \times 2$ & \\
\hline & $0.006149 \times 2$ & $0.004153 \times 2$ & $0.004119 \times 2$ & \\
\hline & $0.993649 \times 2$ & $0.995837 \times 2$ & $0.995879 \times 2$ & $1 \times 4$ \\
\hline & $0.998959 \times 2$ & $0.999352 \times 2$ & $0.999357 \times 2$ & \\
\hline \multirow[t]{10}{*}{ Be, ${ }^{3} S$, triple- $\zeta$} & 0.000004 & 0.000000 & 0.000000 & $0 \times 6$ \\
\hline & 0.000187 & 0.000001 & 0.000000 & \\
\hline & 0.000573 & 0.000011 & 0.000007 & \\
\hline & 0.000645 & 0.000013 & 0.000009 & \\
\hline & 0.000702 & 0.000707 & 0.000707 & \\
\hline & 0.001511 & 0.000712 & 0.000711 & \\
\hline & 0.998534 & 0.999280 & 0.999284 & $1 \times 4$ \\
\hline & 0.999252 & 0.999286 & 0.999287 & \\
\hline & 0.999293 & 0.999990 & 0.999995 & \\
\hline & 0.999299 & 0.999998 & 1.000000 & \\
\hline \multirow[t]{7}{*}{$\mathrm{H}_{2} \mathrm{O},{ }^{1} A_{1}$, STO-6G } & $0.029795 \times 2$ & $0.013850 \times 2$ & $0.013304 \times 2$ & $0 \times 4$ \\
\hline & $0.031585 \times 2$ & $0.014766 \times 2$ & $0.013509 \times 2$ & \\
\hline & $0.970205 \times 2$ & $0.986433 \times 2$ & $0.986732 \times 2$ & $1 \times 10$ \\
\hline & $0.970570 \times 2$ & $0.987475 \times 2$ & $0.988323 \times 2$ & \\
\hline & $0.998733 \times 2$ & $0.998702 \times 2$ & $0.998973 \times 2$ & \\
\hline & $0.999114 \times 2$ & $0.998776 \times 2$ & $0.999161 \times 2$ & \\
\hline & $0.999998 \times 2$ & $0.999999 \times 2$ & $0.999999 \times 2$ & \\
\hline \multirow[t]{7}{*}{$\mathrm{CH}_{2},{ }^{1} A_{1}$, STO-6G } & $0.037796 \times 4$ & $0.014336 \times 2$ & $0.010854 \times 2$ & $0 \times 6$ \\
\hline & $0.314251 \times 2$ & $0.016294 \times 2$ & $0.012979 \times 2$ & \\
\hline & $0.685804 \times 2$ & $0.069501 \times 2$ & $0.050589 \times 2$ & \\
\hline & $0.962204 \times 4$ & $0.929480 \times 2$ & $0.947809 \times 2$ & $1 \times 8$ \\
\hline & $0.999945 \times 2$ & $0.984098 \times 2$ & $0.987470 \times 2$ & \\
\hline & & $0.986297 \times 2$ & $0.990307 \times 2$ & \\
\hline & & $0.999993 \times 2$ & $0.999993 \times 2$ & \\
\hline
\end{tabular}

ues of 1-RDM) for $\operatorname{Be}\left({ }^{1} S\right.$, STO-6G), $\operatorname{Be}\left({ }^{1} S\right.$, triple- $\left.\zeta\right)$, $\mathrm{Be}\left({ }^{3} S\right.$, triple- $\left.\zeta\right), \quad \mathrm{H}_{2} \mathrm{O}\left({ }^{1} A_{1}\right.$, STO-6G), and $\mathrm{CH}_{2}\left({ }^{1} A_{1}\right.$, STO-6G). For Be $\left({ }^{1} S\right.$, STO-6G), the occupation numbers of the $2 p$ orbitals should be sixfold degenerate. Although we did not impose such constraints, this degeneracy accurately holds in both $\mathrm{DM}(P+Q)$ and $\mathrm{DM}(P+Q+G)$ calculations. For singlet states, both $\mathrm{DM}(P+Q)$ and $\mathrm{DM}(P+Q+G)$ calculations reproduced the degeneracy of the twofold occupation without constraints. Generally, the occupation numbers of the DM calculations are much more distributed over all the natural orbitals than those of the full CI. Although such a trend is reduced for the $\operatorname{DM}(P+Q+G)$ calculation, it contradicts our expectation; the occupation numbers are ex- pected to be less distributed in the calculations with less sufficient $N$-representability conditions. An extreme case was $\mathrm{CH}_{2}$, this tendency is very amplified and the accidental degeneracy of occupation are found in the $\mathrm{DM}(P+Q)$ calculation.

In Table VII, the root-mean-square (rms) deviation $d$ of the 2-RDM from the full CI,

$$
d=\sqrt{\sum_{i_{1} i_{2} j_{1} j_{2}}\left\{\left(\Gamma_{\text {calculated }}\right)_{j_{1} j_{2}}^{i_{1} i_{2}}-\left(\Gamma_{\text {full Cl }}\right)_{j_{1} j_{2}}^{i_{1} i_{2}}\right\}^{2}}
$$

is presented for the systems examined in Table VI. The deviations of the 2-RDM are quite small in $\mathrm{DM}(P+Q+G)$

TABLE VII. RMS deviations of the 2-RDMs calculated by DMVT with $P+Q$ and $P+Q+G$ conditions from those by full $\mathrm{CI}$ for $\mathrm{Be}, \mathrm{H}_{2} \mathrm{O}$, and $\mathrm{CH}_{2}$.

\begin{tabular}{lcccc}
\hline \hline System, state, basis & $\mathrm{DM}(P+Q)$ & $\mathrm{DM}(P+Q+G)$ & Full CI & Hartree-Fock \\
\hline $\mathrm{Be},{ }^{1} S$, STO-6G & 0.049208 & 0.000162 & 0 & 0.526569 \\
$\mathrm{Be},{ }^{1} S$, triple- $\zeta$ & 0.049615 & 0.003567 & 0 & 0.100331 \\
$\mathrm{Be},{ }^{3} S$, triple- $\zeta$ & 0.029401 & 0.000715 & 0 & 0.039123 \\
$\mathrm{H}_{2} \mathrm{O},{ }^{1} A_{1}$, STO-6G & 0.467084 & 0.029694 & 0 & 0.266154 \\
$\mathrm{CH}_{2},{ }^{1} A_{1}$, STO-6G & 1.604712 & 0.153503 & 0 & 0.484788 \\
\hline \hline
\end{tabular}


TABLE VIII. Comparison of largest eigenvalues $P, Q, G$-matrices and smallest $G$-matrix calculated by DMVT with $P+Q$ and $P+Q+G$ conditions compared with those obtained by the wave function methods, full CI, and Hartree-Fock for $\mathrm{Be}, \mathrm{H}_{2} \mathrm{O}$, and $\mathrm{CH}_{2}$.

\begin{tabular}{|c|c|c|c|c|}
\hline $\begin{array}{l}\text { System, state, } \\
\text { basis }\end{array}$ & $\mathrm{DM}(P+Q)$ & $\mathrm{DM}(P+Q+G)$ & Full CI & Hartree-Fock \\
\hline \multicolumn{5}{|l|}{$\mathrm{Be},{ }^{1} S$, STO-6G } \\
\hline Largest $P$ & 1.005284 & 1.000907 & 1.000874 & 1 \\
\hline Largest $Q$ & 2.264502 & 2.259861 & 2.259834 & 2 \\
\hline Largest $G$ & 3.675970 & 3.682340 & 3.682407 & 4 \\
\hline Smallest $G$ & -0.007288 & 0.000000 & 0.000000 & 0 \\
\hline \multicolumn{5}{|l|}{ Be, ${ }^{1} S$, triple $-\zeta$} \\
\hline Largest $P$ & 1.004751 & 1.000672 & 1.000612 & 1 \\
\hline Largest $Q$ & 2.008541 & 2.002209 & 2.002072 & 2 \\
\hline Largest $G$ & 3.978475 & 3.985686 & 3.985726 & 4 \\
\hline Smallest $G$ & -0.002482 & 0.000000 & 0.000000 & 0 \\
\hline \multicolumn{5}{|l|}{ Be, ${ }^{3} S$, triple $-\zeta$} \\
\hline Largest $P$ & 1.002019 & 0.999991 & 0.999995 & 1 \\
\hline Largest $Q$ & 2.005387 & 2.000230 & 2.000233 & 2 \\
\hline Largest $G$ & 3.994883 & 3.997833 & 3.997849 & 4 \\
\hline Smallest $G$ & -0.001439 & 0.000000 & 0.000000 & 0 \\
\hline \multicolumn{5}{|c|}{$\mathrm{H}_{2} \mathrm{O},{ }^{1} A_{1}$, STO-6G } \\
\hline Largest $P$ & 1.034378 & 1.010041 & 1.008652 & 1 \\
\hline Largest $G$ & 9.805708 & 9.900236 & 9.904312 & 10 \\
\hline Smallest $G$ & -0.188367 & 0.000000 & 0.000000 & 0 \\
\hline \multicolumn{5}{|c|}{$\mathrm{CH}_{2},{ }^{1} A_{1}$, STO-6G } \\
\hline Largest $P$ & 1.090030 & 1.024402 & 1.019529 & 1 \\
\hline Largest $Q$ & 2.227026 & 2.059546 & 2.042417 & 2 \\
\hline Largest $G$ & 6.942144 & 7.679238 & 7.746013 & 8 \\
\hline Smallest $G$ & -0.208966 & 0.000001 & 0.000000 & 0 \\
\hline
\end{tabular}

calculation especially for small systems, where $\operatorname{DM}(P+Q$ $+G)$ give the identical total energy and virial coefficient to full CI. However, $\mathrm{DM}(P+Q)$ calculations gave worse results and even worse than Hartree-Fock for $\mathrm{H}_{2} \mathrm{O}$ and $\mathrm{CH}_{2}$.

In Table VIII, we compare the largest eigenvalues of $P$, $Q$, and $G$-matrices and smallest eigenvalues of $G$-matrix, for the same systems. Largest eigenvalues of $P$ and $Q$-matrices become smaller as the calculation quality becomes better, while those of $G$-matrix become larger. In $\mathrm{DM}(P+Q)$ calculations, smallest eigenvalues of $G$-matrix are negative. As we expected, smallest eigenvalue of $G$-matrix becomes smaller when electron correlation gets larger. We did not show the smallest eigenvalues of $P$ and $Q$-matrices since in any case, they are almost zero (absolute values are smaller than $\left.10^{-6}\right)$. The deviation of these values that are large for the $\mathrm{CH}_{2}$ [largest eigenvalue of $G$-matrix for $\operatorname{DM}(P+Q$ $+G)$ ] calculation is 7.679238 compared to the full CI's one 7.746013 , while the SDPA errors are small (primal and dual feasibilities are $4.14 \times 10^{-12}$ and $3.54 \times 10^{-6}$, respectively, and gap is $2.69 \times 10^{-7}$ ). Therefore, we conclude that the error originates from the insufficiency of the $N$-representability conditions rather than that of the SDPA.

The trace of the $Q$ matrix is normalized to $(r-N) \times(r$ $-N+1$ ), where $r$ is number of MO (or rank of 1-RDM) and $N$ is the number of the electrons. This condition is satisfied when we impose the constraint for the number of the electrons.

Lastly, we note that we find essentially no problem in finding the minimum and this should be the case for other systems. This is certainly a merit of the present method.

\section{CONCLUSION}

The DMVT is developed systematically by using SDPA as a problem solver. This technique is very stable and there were no example where we could not get a convergence. In addition to several trivial conditions, the $P+Q$ condition is insufficient, while the $P+Q+G$ condition gives satisfactory results, the extent of overshooting the full-CI energy being small for the systems presently examined. The dipole moment and the virial coefficient calculated by the $\operatorname{DM}(P+Q$ $+G)$ method are also very close to the full-CI values. This method is applicable to the ground state of any spin- and space symmetry of closed and open-shell systems.

In this DMVT approach, the calculated energy is a lower bound of the exact energy. The errors of the present $\operatorname{DM}(P$ $+Q+G)$ method are permissible in both energy and properties. Though most quantum chemical method available give the ground-state energy higher than the full-CI one, the present method giving lower energy is equally permissible as an approximate quantum chemical method, if it is stable and feasible in cost performance. For the second requirement, the present stage of the theory is an infant stage, but much progress is expected in future.

\section{ACKNOWLEDGMENT}

This study has been supported by the Grand-in-Aid for Scientific Research from the Japanese Ministry of Education, Science, Culture, and Sports.

${ }^{1}$ K. Husimi, Proc. Phys. Math. Soc. Jpn. 22, 264 (1940).

${ }^{2}$ P.-O. Löwdin, Phys. Rev. 99, 1474 (1955).

${ }^{3}$ H. Nakatsuji, in Many-Electron Densities and Reduced Density Matrices, edited by J. Cioslowski (Kluwer Academic, New York, 2000).

${ }^{4}$ H. Nakatsuji, Phys. Rev. A 14, 41 (1976).

${ }^{5}$ H. Nakatsuji, Theor. Chem. Acc. 102, 97 (1999).

${ }^{6}$ H. Nakatsuji and K. Yasuda, Phys. Rev. Lett. 76, 1039 (1996).

${ }^{7}$ K. Yasuda and H. Nakatsuji, Phys. Rev. A 56, 2648 (1997).

${ }^{8}$ M. Ehara, M. Nakata, H. Kou, K. Yasuda, and H. Nakatsuji, Chem. Phys. Lett. 305, 483 (1999).

${ }^{9}$ M. Nakata, M. Ehara, K. Yasuda, and H. Nakatsuji, J. Chem. Phys. 112, 8772 (2000)

${ }^{10}$ A. J. Coleman, Rev. Mod. Phys. 35, 668 (1963).

${ }^{11}$ C. Garrod and J. Percus, J. Math. Phys. 5, 1756 (1964).

${ }^{12}$ C. Garrod and M. A. Fusco, Int. J. Quantum Chem. x, 495 (1976).

${ }^{13}$ C. Garrod, M. V. Mihailović, and M. Rosina, J. Math. Phys. 16, 868 (1975).

${ }^{14}$ M. V. Mihailović and M. Rosina, Nucl. Phys. A 237, 229 (1975).

${ }^{15}$ K. Fujisawa, M. Kojima, K. Nakata, SDPA (SemiDefinite Programming Algorithm) User's Manual Version 5.00, August 1999, ftp:// ftp.is.titech.ac.jp/pub/OpRes/software/SDPA/5.00/.

${ }^{16}$ R. M. Erdahl and C. Garrod, in Proceedings of the Conference on Density Matrices, Kingston, June 1974, edited by A. J. Coleman and R. M. Erdahl.

${ }^{17}$ H. Kummer, J. Math. Phys. 8, 2063 (1967).

${ }^{18}$ Yu. Nesterov and A. S. Nemirovskii, Interior Point Polynomial Method in Convex Programming: Theory and Applications (SIAM, Philadelphia, 1993).

${ }^{19}$ M. Kojima, Semidefinite Programming and Interior-Point Methods, http://www.is.titech.ac.jp/ kojima/wabun.html, 1996 (in Japanese).

${ }^{20}$ S. Huzinaga, J. Chem. Phys. 42, 1293 (1965).

${ }^{21}$ T. H. Dunning, Jr., J. Chem. Phys. 53, 2823 (1970); T. H. Dunning, Jr. and P. J. Hay, in Method of Electronic Structure Theory, edited by H. F. Schaefer III (Plenum, New York, 1977), Vol. 2; Actually, basis sets are 
taken from EMSL Gaussian Basis Set Order Form, http:// www.emsl.pnl.gov:2080/forms/basisform.html.

${ }^{22}$ W. J. Hehre, R. F. Stweart, and J. A. Pople, J. Chem. Phys. 51, 2657 (1969).

${ }^{23}$ K. P. Huber and G. Herzberg, Molecular Spectra and Molecular Structure
IV, Electronic Constants of Diatomic Molecules (Van Nostrand Reinhold, New York, 1979).

${ }^{24}$ J. H. Callomon, E. Horita, K. Kuchitsu, W. J. Lafferty, A. G. Maki, and C.S. Pote, Landolt-Börnstein (Springer-Verlag, Berlin, 1976).

${ }^{25}$ A. J. Coleman, Rep. Math. Phys. 4, 113 (1973). 